Indications of Suppression of Excited Y States in Pb-Pb Collisions at $\sqrt{s_{\mathrm{NN}}}=2.76 \mathrm{TeV}$

\author{
S. Chatrchyan et al. ${ }^{*}$ \\ (CMS Collaboration)
}

(Received 25 May 2011; published 28 July 2011)

\begin{abstract}
A comparison of the relative yields of $Y$ resonances in the $\mu^{+} \mu^{-}$decay channel in $\mathrm{Pb}-\mathrm{Pb}$ and $p p$ collisions at a center-of-mass energy per nucleon pair of $2.76 \mathrm{TeV}$ is performed with data collected with the CMS detector at the LHC. Using muons of transverse momentum above $4 \mathrm{GeV} / c$ and pseudorapidity below 2.4, the double ratio of the $\mathrm{Y}(2 S)$ and $\mathrm{Y}(3 S)$ excited states to the $\mathrm{Y}(1 S)$ ground state in $\mathrm{Pb}-\mathrm{Pb}$ and $p p$ collisions, $[\mathrm{Y}(2 S+3 S) / \mathrm{Y}(1 S)]_{\mathrm{Pb}-\mathrm{Pb}} /[\mathrm{Y}(2 S+3 S) / \mathrm{Y}(1 S)]_{p p}$, is found to be $0.31_{-0.15}^{+0.19}$ (stat) \pm 0.03 (syst). The probability to obtain the measured value, or lower, if the true double ratio is unity, is calculated to be less than $1 \%$.
\end{abstract}

DOI: 10.1103/PhysRevLett.107.052302

PACS numbers: 25.75.Nq, 14.40.Pq

Quantum chromodynamics (QCD) predicts that strongly interacting matter undergoes a phase transition to a deconfined state, often referred to as the quark-gluon plasma (QGP), in which quarks and gluons are no longer bound within hadrons. Calculations in lattice QCD [1,2] indicate that the transition should occur at a critical temperature $T_{c} \simeq 150-175 \mathrm{MeV}$, corresponding to an energy density $\varepsilon_{c} \simeq 1 \mathrm{GeV} / \mathrm{fm}^{3}$.

If the QGP is formed in heavy-ion collisions, it is expected to screen the confining potential of heavy quark-antiquark pairs [3], leading to the melting of charmonia $\left(J / \psi, \psi^{\prime}, \chi_{c} \ldots\right)$ and bottomonia $(\Upsilon(1 S), \Upsilon(2 S)$, $\left.\Upsilon(3 S), \chi_{b} \ldots\right)$. The melting temperature depends on the binding energy of the quarkonium state. The ground states $J / \psi$, and $Y(1 S)$, are expected to dissolve at significantly higher temperatures than the more loosely bound excited states. Quenched lattice QCD calculations [4,5] predict that the $Y(n S)$ states melt at $1.2 T_{c}(3 S), 1.6 T_{c}(2 S)$, and above $4 T_{c}(1 S)$, while modern spectral-function approaches with complex potentials [6,7] favor somewhat lower dissolution temperatures. This sequential melting pattern is generally considered a "smoking-gun" signature of the QCD deconfinement transition. However, a large fraction of the observed $Y(1 S)$ yield is due to decays of heavier states (around $50 \%$ for the $Y(1 S)$ [8]). Therefore the melting of the excited states is expected to result in a significant suppression of the observed $\Upsilon(1 S)$ yield, even if the medium is not hot enough to directly dissolve it.

Observations of a larger $J / \psi$ and $\psi(2 S)$ suppression in heavy-ion collisions with respect to proton-nucleus collisions were reported by the NA38 [9], NA50 [10,11], and NA60 [12] fixed-target experiments at the Super Proton

*Full author list given at the end of the article.

Published by the American Physical Society under the terms of the Creative Commons Attribution 3.0 License. Further distribution of this work must maintain attribution to the author(s) and the published article's title, journal citation, and DOI.
Synchrotron (SPS), respectively, in sulfur-uranium, leadlead, and indium-indium collisions, at center-of-mass energies per nucleon pair $\left(\sqrt{s_{\mathrm{NN}}}\right)$ of about $20 \mathrm{GeV}$. The PHENIX experiment, at the Relativistic Heavy Ion Collider (RHIC), extended the $J / \psi$ suppression measurements to $\mathrm{Au}-\mathrm{Au}$ collisions at $\sqrt{s_{\mathrm{NN}}}=200 \mathrm{GeV}$ [13]. Recent results from ATLAS and CMS show $J / \psi$ suppression at LHC $[14,15]$. At RHIC, bottomonia production becomes measurable [16], though with limited integrated luminosities. PHENIX observed that the dimuon yield in the $\mathrm{Y}$ mass region for minimum bias $\mathrm{Au}-\mathrm{Au}$ collisions is less than $64 \%$, at the $90 \%$ confidence level, of the value expected by extrapolating the proton-proton yields [17].

A new era of detailed studies of the bottomonium family in heavy-ion collisions has started at the Large Hadron Collider (LHC). The measurement reported in this Letter is performed with data recorded by the Compact Muon Solenoid (CMS) experiment during the first lead-lead $(\mathrm{Pb}-\mathrm{Pb})$ LHC run, at the end of 2010, and during the proton-proton $(p p)$ run of March 2011, both at $\sqrt{s_{\mathrm{NN}}}=$ $2.76 \mathrm{TeV}$. The integrated luminosity used in this analysis corresponds to $7.28 \mu \mathrm{b}^{-1}$ for $\mathrm{Pb}-\mathrm{Pb}$ and $225 \mathrm{nb}^{-1}$ for $p p$ collisions, the latter corresponding approximately to the equivalent nucleon-nucleon luminosity of the $\mathrm{Pb}-\mathrm{Pb}$ run. The momentum resolution of the CMS detector results in well-resolved $Y$ peaks in the dimuon mass spectrum. The CMS Collaboration has previously studied $Y$ production in $p p$ data at $\sqrt{s}=7 \mathrm{TeV}$ [18], using techniques to extract the $Y$ yields that are very similar to the ones used in the study reported in this Letter.

A detailed description of the CMS detector can be found in [19]. Its central feature is a superconducting solenoid of $6 \mathrm{~m}$ internal diameter, providing a magnetic field of 3.8 T. Within the field volume are the silicon $(\mathrm{Si})$ pixel and strip tracker, the crystal electromagnetic calorimeter, and the brass or scintillator hadron calorimeter. Muons are measured in gas-ionization detectors embedded in the steel return yoke. In addition, CMS has extensive 
forward calorimetry, in particular, two steel or quartz-fiber Čerenkov hadron forward (HF) calorimeters, which cover the pseudorapidity range $2.9<|\eta|<5.2$.

In this analysis, $Y$ states are identified through their dimuon decay. The silicon pixel and strip tracker measures charged-particle trajectories in the range $|\eta|<2.5$. The tracker consists of $66 \mathrm{M}$ pixel and $10 \mathrm{M}$ strip detector channels, providing a vertex resolution of $\sim 15 \mu \mathrm{m}$ in the transverse plane. Muons are detected in the $|\eta|<2.4$ range, with detection planes based on three technologies: drift tubes, cathode strip chambers, and resistive plate chambers. Because of the strong magnetic field and the fine granularity of the $\mathrm{Si}$ tracker, the muon transverse momentum measurement $\left(p_{T}\right)$ based on information from the Si tracker alone has a resolution between $1 \%$ and $2 \%$ for a typical muon in this analysis.

In both the $\mathrm{Pb}-\mathrm{Pb}$ and $p p$ runs, the events are selected by the CMS two-level trigger. At the first, hardware level, two independent muon candidates are required in the muon detectors. No selection is made on momentum or pseudorapidity, but in the $p p$ case more stringent quality requirements are imposed for each muon in order to reduce the higher trigger rate. In both cases, the software-based higher-level trigger accepts the lower-level decision without applying further criteria. The single-muon trigger efficiencies are measured from reconstructed $J / \psi \rightarrow \mu \mu$ decays, for muons with $p_{T}>4 \mathrm{GeV} / c$. The values of these efficiencies, $(96.1 \pm 1.0) \%$ in the $\mathrm{Pb}-\mathrm{Pb}$ data set and $(95.5 \pm 0.6) \%$ in the $p p$ data set, are consistent.

For the $\mathrm{Pb}-\mathrm{Pb}$ data, events are preselected offline if they contain a reconstructed primary vertex made of at least two tracks, and a coincidence in both HF calorimeters of energy deposits larger than $3 \mathrm{GeV}$ in at least three towers. These criteria reduce contributions from singlebeam interactions (e.g., beam-gas and beam-halo collisions with the beam pipe), ultraperipheral electromagnetic collisions, and cosmic-ray muons. A small fraction of the most peripheral $\mathrm{Pb}-\mathrm{Pb}$ collisions is not selected by these requirements, which accept $(97 \pm 3) \%$ of the hadronic inelastic cross section [20]. For the $p p$ run, a similar event filter is applied, relaxing the HF coincidence to one tower in each HF, with at least $3 \mathrm{GeV}$ deposited. This filter removes only $1 \%$ of the $p p$ events satisfying the dimuon trigger.

The muon offline reconstruction is seeded with $\simeq 99 \%$ efficiency by tracks in the muon detectors, called standalone muons. These tracks are then matched to tracks reconstructed in the Si tracker by means of an algorithm optimized for the heavy-ion environment [21,22]. For muons from $Y$ decays the Si-tracking efficiency is $\simeq 85 \%$. This efficiency is lower than in $p p$, as in $\mathrm{Pb}-\mathrm{Pb}$ the Si-track reconstruction is seeded by a greater number of pixel hits to reduce the large number of random combinations arising from the high multiplicity of each event. Combined fits of the muon and Si-tracker tracks are used to obtain the results presented in this Letter. The heavy-ion dedicated reconstruction algorithm is applied to the $p p$ data in order to avoid potential biases.

Identical, very-loose selection criteria are applied to the muons in the $p p$ and $\mathrm{Pb}-\mathrm{Pb}$ data. Their transverse (longitudinal) distance of closest approach from the event vertex is required to be less than $3(15) \mathrm{cm}$. Tracks are only kept if they have 11 or more hits in the silicon tracker and the $\chi^{2}$ per degree of freedom of the combined (tracker) track fit is lower than 20 (4). The two muon trajectories are fit with a common vertex constraint, and events are retained if the fit $\chi^{2}$ probability is larger than $1 \%$. This removes background arising primarily from the displaced, semileptonic decays of charm and bottom hadrons. As determined from Monte Carlo simulation of the $Y(1 S)$ signal, these selection criteria are found to reduce the efficiency by $3.9 \%$, which is consistent with the signal loss observed in both $p p$ and $\mathrm{Pb}-$ $\mathrm{Pb}$ data. The available event sample limits to $20 \mathrm{GeV} / c$ the dimuon transverse momentum range probed in this study.

Only muons with a transverse momentum $\left(p_{T}^{\mu}\right)$ higher than $4 \mathrm{GeV} / c$ are considered, resulting in a $Y$ acceptance of approximately $25 \%$ for the $\left|y^{\Upsilon}\right|<2.4$ rapidity range. This requirement optimizes the significance of the $Y(1 S)$ signal in $\mathrm{Pb}-\mathrm{Pb}$ data and is applied to both data sets. The acceptance of a $Y$ state depends on its mass, since the excited states give rise to higher-momenta muons. In consequence, requiring higher $p_{T}^{\mu}$ increases the acceptance for the excited states relative to the ground state. In the corresponding analysis performed with the higher statistics $\left(3.1 \mathrm{pb}^{-1}\right) 7 \mathrm{TeV}$ data [18], looser criteria were applied $\left(p_{T}^{\mu}>3.5 \mathrm{GeV} / c\right.$ and $\left|\eta^{\mu}\right|<1.6$, or $p_{T}^{\mu}>2.5 \mathrm{GeV} / c$ and $1.6<\left|\eta^{\mu}\right|<2.4$ ), where $\eta^{\mu}$ is the muon pseudorapidity. The stricter $\left(p_{T}^{\mu}>4 \mathrm{GeV} / c\right)$ requirements used here enhance the $\Upsilon(2 S+3 S) / \Upsilon(1 S)$ yield ratio by $\simeq 60 \%$ in the $p p$ data at $2.76 \mathrm{TeV}$. It was checked that, applying identical $p p$ reconstruction algorithms and $p_{T}^{\mu}$ requirements, the $Y(2 S+3 S) / \Upsilon(1 S)$ yield ratio is consistent, within statistical uncertainties, between the 2.76 and $7 \mathrm{TeV} p p$ data sets [15].

The dimuon invariant-mass spectra with the selection criteria applied are shown in Fig. 1 for the $p p$ and $\mathrm{Pb}-\mathrm{Pb}$ data sets. Within the $7-14 \mathrm{GeV} / c^{2}$ mass range, there are 561 (628) opposite-sign muon pairs in the $p p(\mathrm{~Pb}-\mathrm{Pb})$ data set. The three $Y$ peaks are clearly observed in the $p p$ case, but the $Y(2 S)$ and $Y(3 S)$ are not visible over the residual background in $\mathrm{Pb}-\mathrm{Pb}$ collisions.

An extended unbinned maximum likelihood fit to the two invariant-mass distributions of Fig. 1 is performed to extract the yields, following the method described in [18]. The measured mass line shape of each $Y$ state is parametrized by a "crystal ball" (CB) function, i.e., a Gaussian resolution function with the lowside tail replaced by a power law describing final-state radiation (FSR). Since the three $Y$ resonances partially overlap in the measured dimuon mass, they are fit simultaneously. Therefore, the 

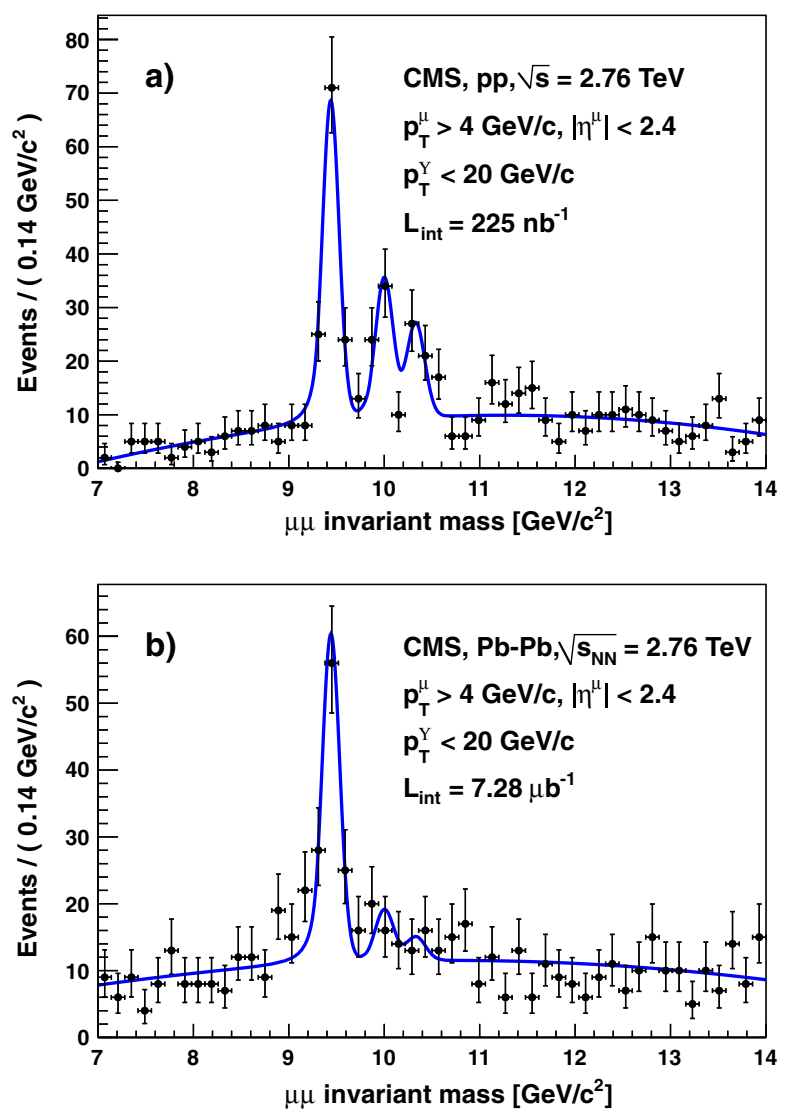

FIG. 1 (color online). Dimuon invariant-mass distributions from the $p p$ (a) and $\mathrm{Pb}-\mathrm{Pb}(\mathrm{b})$ data at $\sqrt{s_{\mathrm{NN}}}=2.76 \mathrm{TeV}$. The same reconstruction algorithm and analysis criteria are applied to both data sets, including a transverse momentum requirement on single muons of $p_{T}^{\mu}>4 \mathrm{GeV} / c$. The solid lines show the result of the fit described in the text.

probability distribution function (PDF) describing the signal consists of three $\mathrm{CB}$ functions. In addition to the three $\Upsilon(n S)$ yields, the $\Upsilon(1 S)$ mass is the only parameter left free, to accommodate a possible bias in the momentum scale calibration. The mass ratios between the states are fixed to their world average values [23] and the mass resolution is forced to scale with the resonance mass. The $Y(1 S)$ resolution is fixed to the value estimated in the simulation, $92 \mathrm{MeV} / c^{2}$, which is compatible with the resolution obtained from both the $\mathrm{Pb}-\mathrm{Pb}$ and $p p$ data. The lowside tail parameters are also fixed to the values obtained via simulation. Finally, a second-order polynomial is chosen to describe the background in the $7-14 \mathrm{GeV} / c^{2}$ mass-fit range.

The quality of the unbinned fit is checked a posteriori by comparing the obtained line shapes to the binned data of Fig. 1. The $\chi^{2}$ probabilities are $74 \%$ and $77 \%$, respectively, for $p p$ and $\mathrm{Pb}-\mathrm{Pb}$.

The ratios of the observed (uncorrected) yields of the $\Upsilon(2 S)$ and $\Upsilon(3 S)$ excited states to the $\Upsilon(1 S)$ ground state in the $p p$ and $\mathrm{Pb}-\mathrm{Pb}$ data are

$$
\begin{gathered}
\Upsilon(2 S+3 S) /\left.\Upsilon(1 S)\right|_{p p}=0.78_{-0.14}^{+0.16} \pm 0.02, \\
\Upsilon(2 S+3 S) /\left.\Upsilon(1 S)\right|_{\mathrm{Pb}-\mathrm{Pb}}=0.24_{-0.12}^{+0.13} \pm 0.02,
\end{gathered}
$$

where the first uncertainty is statistical and the second is systematic.

The systematic uncertainties are computed by varying the line shape in the following ways: (1) the CB-tail parameters are varied randomly according to their covariance matrix and within conservative values covering imperfect knowledge of the amount of detector material and FSR in the underlying process; (2) the resolution is varied by $\pm 5 \mathrm{MeV} / c^{2}$, which is a conservative variation given the current understanding of the detector performance and reasonable changes that can be anticipated in the $Y$-resonance kinematics between $p p$ and $\mathrm{Pb}-\mathrm{Pb}$ data; (3) the background shape is changed from quadratic to linear while the mass range of the fit is varied from 6-15 to $8-12 \mathrm{GeV} / c^{2}$; the observed root-mean-square of the results is taken as the systematic uncertainty. The quadratic sum of these three systematic uncertainties gives a relative uncertainty on the ratio of $10 \%(3 \%)$ for the $\mathrm{Pb}-\mathrm{Pb}$ $(p p)$ data.

The ratio of the $\Upsilon(2 S+3 S) / \Upsilon(1 S)$ ratios in $\mathrm{Pb}-\mathrm{Pb}$ and $p p$ benefits from an almost complete cancellation of possible acceptance and/or efficiency differences among the reconstructed resonances. A simultaneous fit to the $p p$ and $\mathrm{Pb}-\mathrm{Pb}$ mass spectra gives the double ratio

$$
\frac{\Upsilon(2 S+3 S) /\left.\mathrm{Y}(1 S)\right|_{\mathrm{Pb}-\mathrm{Pb}}}{\mathrm{Y}(2 S+3 S) /\left.\mathrm{Y}(1 S)\right|_{p p}}=0.31_{-0.15}^{+0.19}(\text { stat }) \pm 0.03 \text { (syst) }
$$

where the systematic uncertainty (9\%) arises from varying the line shape as described above in the simultaneous fit, thus taking into account partial cancellations of systematic effects.

The single-muon lower momentum requirement is a posteriori varied from 3 to $5 \mathrm{GeV} / c$ in steps of $500 \mathrm{MeV} / c$, and it is found that $p_{T}$ requirements other than $4 \mathrm{GeV} / c$ produce lower values of the double ratio. Fitting the $p p$ and $\mathrm{Pb}-\mathrm{Pb}$ spectra with free and independent mass resolution parameters leads to an increase of the double ratio by $15 \%$.

To evaluate possible imperfect cancellations of acceptance and efficiency effects in the double ratio, a full [24] detector simulation is performed. The effect of the higher $\mathrm{Pb}-\mathrm{Pb}$ underlying event activity is accounted for by embedding, at the level of detector signals, $\Upsilon(1 S)$ and $\Upsilon(2 S)$ decays simulated by PYTHIA 6.424 [25] in $\mathrm{Pb}-\mathrm{Pb}$ events simulated with HYDJET [26]. Track characteristics, such as the number of hits and the $\chi^{2}$ of the track fit, have similar distributions in data and simulation. As mentioned above, the trigger efficiency is evaluated with data, by using single-muon-triggered data events, and reconstructing $J / \psi$ signal with and without the dimuon trigger 
requirement. The same exercise is carried out with the simulation and it agrees with the efficiency measured in data at the $2 \%$ level. The track efficiency in the silicon detector is measured with standalone muons, applying all selection criteria. The efficiencies in data and simulation agree within the $4 \%$ statistical uncertainty of the efficiency determined from data.

The difference in reconstruction and selection efficiencies between the $Y$ states is less than 5\% and the relative variation with charged-particle multiplicity is about $10 \%$ from $p p$ to central $\mathrm{Pb}-\mathrm{Pb}$ collisions, producing a maximum change of $0.5 \%$ on the double ratio. The good agreement between single-muon trigger efficiencies extracted from data for the $p p$ and $\mathrm{Pb}-\mathrm{Pb}$ trigger requirements, applied to the $Y(1 S)$ and $Y(2 S)$ trigger efficiencies derived from simulation, leads to a negligible effect on the double ratio. The single-muon trigger efficiencies extracted from data agree within $1.5 \%$ for the $p p$ and $\mathrm{Pb}-\mathrm{Pb}$ trigger requirements, and the $\Upsilon(1 S)$ and $\Upsilon(2 S)$ trigger efficiencies agree within 3\%, according to simulation: the potential trigger bias on the double ratio is negligible. Even doubling the size of these variations, to take the $Y(3 S)$ into account, leads to a negligible change in the double ratio. The magnitudes of the statistical and systematic uncertainties on the double ratio, respectively, 55\% and 9\%, are significantly larger than the systematic uncertainties associated with possible imperfect cancellation of acceptance and efficiency effects. Therefore no additional uncertainty from these sources is applied.

Finally, using an ensemble of $1 \times 10^{6}$ pseudoexperiments, generated with the signal line shape obtained from the $p p$ data [Fig. 1(a)], the background line shapes from both data sets, and a double ratio [Eq. (3)] equal to unity within statistical and systematic uncertainties, the probability of finding the measured value of 0.31 or below is estimated to be $0.9 \%$. In other words, in the absence of a suppression due to physics mechanisms, the probability of a downward departure of the ratio from unity of this significance or greater is $0.9 \%$, i.e., that corresponding to 2.4 sigma in a one-tailed integral of a Gaussian distribution.

Other studies from the CMS experiment show that the $\Upsilon(1 S)$ is suppressed by about $40 \%$ [15] in minimum bias $\mathrm{Pb}-\mathrm{Pb}$ collisions at $\sqrt{s_{\mathrm{NN}}}=2.76 \mathrm{TeV}$. Since a large fraction of the $Y(1 S)$ yield arises from decays of heavier bottomonium states [8], this $Y(1 S)$ suppression could be indirectly caused by the suppression of the excited states reported in this Letter.

Production yields of quarkonium states can also be modified, from $p p$ to $\mathrm{Pb}-\mathrm{Pb}$ collisions, in the absence of QGP formation, by cold nuclear matter effects [27]. However, such effects should have a small impact on the $\Upsilon$ double ratio reported here. The nuclear modifications of the parton distribution functions (shadowing) should have an equivalent effect on the three $Y$ states, because their production involves very similar partons, canceling in the ratio, at least to first order. The same should happen to any other initial-state nuclear effect. In principle, the larger and more loosely bound excited quarkonium states are more likely to be broken up by final-state interactions while traversing the nuclear matter, something extensively studied in the context of charmonium suppression at lower energies [28]. This "nuclear absorption" becomes weaker with increasing energy, and should be negligible at the LHC. At RHIC energies, the STAR experiment [29] has reported a $Y(1 S+2 S+3 S)$ yield in $d$-Au collisions of $0.78 \pm 0.28 \pm 0.20$ times the yield expected by scaling $p p$ collisions, compatible with the absence of absorption. Furthermore, the double ratio presented here would only be sensitive to a difference between the nuclear dependencies of the three states and already at much lower energies the Fermilab E772 experiment observed [30], in protonnucleus collisions, no such difference, within uncertainties, between the $Y(1 S)$ and the sum $Y(2 S+3 S)$.

Future high-statistics heavy-ion and proton-nucleus runs at the LHC will provide further quarkonia measurements, which should help disentangle nuclear from medium effects and aid the interpretation of the result reported in this Letter.

In summary, a comparison of the relative yields of $Y$ resonances has been performed in $\mathrm{Pb}-\mathrm{Pb}$ and $p p$ collisions at the same center-of-mass energy per nucleon pair of $2.76 \mathrm{TeV}$. The double ratio of the $Y(2 S)$ and $Y(3 S)$ excited states to the $\mathrm{Y}(1 S)$ ground state in $\mathrm{Pb}-\mathrm{Pb}$ and $p p$ collisions, $[\mathrm{Y}(2 S+3 S) / \mathrm{Y}(1 S)]_{\mathrm{Pb}-\mathrm{Pb}} /[\mathrm{Y}(2 S+3 S) / \mathrm{Y}(1 S)]_{p p}$, is found to be $0.31_{-0.15}^{+0.19}$ (stat) \pm 0.03 (syst), for muons of $p_{T}>$ $4 \mathrm{GeV} / c$ and $|\eta|<2.4$. The probability to obtain the measured value, or lower, if the true double ratio is unity, has been calculated to be less than $1 \%$.

We wish to congratulate our colleagues in the CERN accelerator departments for the excellent performance of the LHC machine. We thank the technical and administrative staff at CERN and other CMS institutes, and acknowledge support from: FMSR (Austria); FNRS and FWO (Belgium); CNPq, CAPES, FAPERJ, and FAPESP (Brazil); MES (Bulgaria); CERN; CAS, MoST, and NSFC (China); COLCIENCIAS (Colombia); MSES (Croatia); RPF (Cyprus); Academy of Sciences and NICPB (Estonia); Academy of Finland, ME, and HIP (Finland); CEA and CNRS/IN2P3 (France); BMBF, DFG, and HGF (Germany); GSRT (Greece); OTKA an NKTH (Hungary); DAE and DST (India); IPM (Iran); SFI (Ireland); INFN (Italy); NRF and WCU (Korea); LAS (Lithuania); CINVESTAV, CONACYT, SEP, and UASLP-FAI (Mexico); PAEC (Pakistan); SCSR (Poland); FCT (Portugal); JINR (Armenia, Belarus, Georgia, Ukraine, Uzbekistan); MST and MAE (Russia); MSTD (Serbia); MICINN and CPAN (Spain); Swiss Funding Agencies (Switzerland); NSC (Taipei); TUBITAK and TAEK (Turkey); STFC (United Kingdom); DOE and 
NSF (U.S.). Individuals have received support from the Marie-Curie programme and the European Research Council (European Union); the Leventis Foundation; the A.P. Sloan Foundation; the Alexander von Humboldt Foundation; the Associazione per lo Sviluppo Scientifico e Tecnologico del Piemonte (Italy); the Belgian Federal Science Policy Office; the Fonds pour la Formation à la Recherche dans l'industrie et dans l'Agriculture (FRIA-Belgium); and the Agentschap voor Innovatie door Wetenschap en Technologie (IWT-Belgium).

[1] F. Karsch, Lect. Notes Phys. 583, 209 (2002).

[2] S. Borsanyi et al. (Wuppertal-Budapest Collaboration), J. High Energy Phys. 09 (2010) 073.

[3] T. Matsui and H. Satz, Phys. Lett. B 178, 416 (1986).

[4] H. Satz, J. Phys. G 32, R25 (2006).

[5] C.-Y. Wong, Phys. Rev. C 72, 034906 (2005).

[6] A. Mocsy and P. Petreczky, Phys. Rev. Lett. 99, 211602 (2007).

[7] C. Miao, A. Mocsy, and P. Petreczky, Nucl. Phys. A 855, 125 (2011).

[8] A. Affolder et al. (CDF Collaboration), Phys. Rev. Lett. 84, 2094 (2000).

[9] C. Baglin et al. (NA38 Collaboration), Phys. Lett. B 345, 617 (1995).

[10] B. Alessandro et al. (NA50 Collaboration), Eur. Phys. J. C 39, 335 (2005).

[11] B. Alessandro et al. (NA50 Collaboration), Eur. Phys. J. C 49, 559 (2007).

[12] R. Arnaldi et al. (NA60 Collaboration), Phys. Rev. Lett. 99, 132302 (2007).

[13] A. Adare et al. (PHENIX Collaboration), Phys. Rev. Lett. 98, 232301 (2007).
[14] G. Aad et al. (ATLAS Collaboration), Phys. Lett. B 697, 294 (2011).

[15] CMS Collaboration, CMS Physics Analysis Summary, CMS-PAS-HIN-10-006, 2011, http://cdsweb.cern.ch/ record/1353586.

[16] B. I. Abelev et al. (STAR Collaboration), Phys. Rev. D 82, 012004 (2010).

[17] E. T. Atomssa (PHENIX Collaboration), Nucl. Phys. A830, 331c (2009).

[18] V. Khachatryan et al. (CMS Collaboration), Phys. Rev. D 83, 112004 (2011).

[19] S. Chatrchyan et al. (CMS Collaboration), JINST 3, S08004 (2008).

[20] CMS Collaboration, arXiv:1102.1957 [Phys. Rev. C (to be published)].

[21] C. Roland (CMS Collaboration), Nucl. Instrum. Methods Phys. Res., Sect. A 566, 123 (2006).

[22] D. d'Enterria et al. (CMS Collaboration), J. Phys. G 34, 2307 (2007).

[23] K. Nakamura et al. (Particle Data Group), J. Phys. G 37, 075021 (2010).

[24] S. Agostinelli et al. (GEANT4 Collaboration), Nucl. Instrum. Methods Phys. Res., Sect. A 506, 250 (2003).

[25] T. Sjöstrand, S. Mrenna, and P. Skands, J. High Energy Phys. 05 (2006) 026.

[26] I. P. Lokhtin and A. M. Snigirev, Eur. Phys. J. C 45, 211 (2006).

[27] R. Vogt, Phys. Rev. C 81, 044903 (2010).

[28] C. Lourenco, R. Vogt, and H. K. Woehri, J. High Energy Phys. 02 (2009) 014.

[29] R. Reed (STAR Collaboration), J. Phys. Conf. Ser. 270, 012026 (2011).

[30] D. M. Alde et al., Phys. Rev. Lett. 66, 2285 (1991).

S. Chatrchyan, ${ }^{1}$ V. Khachatryan, ${ }^{1}$ A. M. Sirunyan, ${ }^{1}$ A. Tumasyan, ${ }^{1}$ W. Adam, ${ }^{2}$ T. Bergauer, ${ }^{2}$ M. Dragicevic, ${ }^{2}$ J. Erö, ${ }^{2}$ C. Fabjan, ${ }^{2}$ M. Friedl, ${ }^{2}$ R. Frühwirth, ${ }^{2}$ V. M. Ghete, ${ }^{2}$ J. Hammer, ${ }^{2, b}$ S. Hänsel, ${ }^{2}$ M. Hoch,${ }^{2}$ N. Hörmann, ${ }^{2}$ J. Hrubec, ${ }^{2}$ M. Jeitler, ${ }^{2}$ W. Kiesenhofer, ${ }^{2}$ M. Krammer, ${ }^{2}$ D. Liko, ${ }^{2}$ I. Mikulec, ${ }^{2}$ M. Pernicka, ${ }^{2}$ B. Rahbaran, ${ }^{2}$ H. Rohringer, ${ }^{2}$

R. Schöfbeck, ${ }^{2}$ J. Strauss, ${ }^{2}$ A. Taurok, ${ }^{2}$ F. Teischinger, ${ }^{2}$ P. Wagner, ${ }^{2}$ W. Waltenberger, ${ }^{2}$ G. Walzel, ${ }^{2}$ E. Widl, ${ }^{2}$ C.-E. Wulz, ${ }^{2}$ V. Mossolov, ${ }^{3}$ N. Shumeiko, ${ }^{3}$ J. Suarez Gonzalez, ${ }^{3}$ S. Bansal, ${ }^{4}$ L. Benucci, ${ }^{4}$ E. A. De Wolf,,${ }^{4}$ X. Janssen, ${ }^{4}$ J. Maes, ${ }^{4}$ T. Maes, ${ }^{4}$ L. Mucibello, ${ }^{4}$ S. Ochesanu, ${ }^{4}$ B. Roland, ${ }^{4}$ R. Rougny, ${ }^{4}$ M. Selvaggi, ${ }^{4}$ H. Van Haevermaet, ${ }^{4}$ P. Van Mechelen, ${ }^{4}$ N. Van Remortel, ${ }^{4}$ F. Blekman, ${ }^{5}$ S. Blyweert,${ }^{5}$ J. D’Hondt, ${ }^{5}$ O. Devroede,${ }^{5}$ R. Gonzalez Suarez, ${ }^{5}$ A. Kalogeropoulos, ${ }^{5}$ M. Maes, ${ }^{5}$ W. Van Doninck,${ }^{5}$ P. Van Mulders, ${ }^{5}$ G. P. Van Onsem, ${ }^{5}$ I. Villella, ${ }^{5}$ O. Charaf, ${ }^{6}$ B. Clerbaux,${ }^{6}$ G. De Lentdecker, ${ }^{6}$ V. Dero, ${ }^{6}$ A. P. R. Gay, ${ }^{6}$ G. H. Hammad, ${ }^{6}$ T. Hreus, ${ }^{6}$ P.E. Marage, ${ }^{6}$ L. Thomas, ${ }^{6}$ C. Vander Velde, ${ }^{6}$ P. Vanlaer, ${ }^{6}$ V. Adler, ${ }^{7}$ A. Cimmino, ${ }^{7}$ S. Costantini, ${ }^{7}$ M. Grunewald, ${ }^{7}$ B. Klein, ${ }^{7}$ J. Lellouch, ${ }^{7}$ A. Marinov, ${ }^{7}$ J. Mccartin, ${ }^{7}$ D. Ryckbosch, ${ }^{7}$ F. Thyssen, ${ }^{7}$ M. Tytgat, ${ }^{7}$ L. Vanelderen, ${ }^{7}$ P. Verwilligen, ${ }^{7}$ S. Walsh, ${ }^{7}$ N. Zaganidis, ${ }^{7}$ S. Basegmez, ${ }^{8}$ G. Bruno, ${ }^{8}$ J. Caudron, ${ }^{8}$ L. Ceard,${ }^{8}$ E. Cortina Gil, ${ }^{8}$ J. De Favereau De Jeneret,${ }^{8}$ C. Delaere, ${ }^{8}$ D. Favart, ${ }^{8}$ A. Giammanco,${ }^{8}$ G. Grégoire, ${ }^{8}$ J. Hollar, ${ }^{8}$ V. Lemaitre, ${ }^{8}$ J. Liao, ${ }^{8}$ O. Militaru, ${ }^{8}$ C. Nuttens, ${ }^{8}$ S. Ovyn, ${ }^{8}$ D. Pagano, ${ }^{8}$ A. Pin, ${ }^{8}$ K. Piotrzkowski, ${ }^{8}$ N. Schul,${ }^{8}$ N. Beliy, ${ }^{9}$ T. Caebergs, ${ }^{9}$ E. Daubie, ${ }^{9}$ G. A. Alves,${ }^{10}$ L. Brito, ${ }^{10}$ D. De Jesus Damiao, ${ }^{10}$ M.E. Pol, ${ }^{10}$ M. H. G. Souza,${ }^{10}$ W. L. Aldá Júnior, ${ }^{11}$ W. Carvalho, ${ }^{11}$

E. M. Da Costa, ${ }^{11}$ C. De Oliveira Martins, ${ }^{11}$ S. Fonseca De Souza, ${ }^{11}$ L. Mundim, ${ }^{11}$ H. Nogima,${ }^{11}$ V. Oguri, ${ }^{11}$ W. L. Prado Da Silva, ${ }^{11}$ A. Santoro, ${ }^{11}$ S. M. Silva Do Amaral,${ }^{11}$ A. Sznajder, ${ }^{11}$ C. A. Bernardes, ${ }^{12, \mathrm{c}}$ F. A. Dias, ${ }^{12}$ T. R. Fernandez Perez Tomei, ${ }^{12}$ E. M. Gregores,${ }^{12, \mathrm{c}}$ C. Lagana, ${ }^{12}$ F. Marinho, ${ }^{12}$ P. G. Mercadante, ${ }^{12, \mathrm{c}}$ S. F. Novaes, ${ }^{12}$ Sandra S. Padula, ${ }^{12}$ N. Darmenov, ${ }^{13, b}$ V. Genchev, ${ }^{13, b}$ P. Iaydjiev, ${ }^{13, b}$ S. Piperov, ${ }^{13}$ M. Rodozov, ${ }^{13}$ S. Stoykova, ${ }^{13}$ 
G. Sultanov, ${ }^{13}$ V. Tcholakov, ${ }^{13}$ R. Trayanov, ${ }^{13}$ A. Dimitrov,${ }^{14}$ R. Hadjiiska, ${ }^{14}$ A. Karadzhinova, ${ }^{14}$ V. Kozhuharov, ${ }^{14}$ L. Litov, ${ }^{14}$ M. Mateev, ${ }^{14}$ B. Pavlov, ${ }^{14}$ P. Petkov, ${ }^{14}$ J. G. Bian,${ }^{15}$ G. M. Chen,${ }^{15}$ H. S. Chen, ${ }^{15}$ C. H. Jiang,,${ }^{15}$ D. Liang, ${ }^{15}$ S. Liang, ${ }^{15}$ X. Meng, ${ }^{15}$ J. Tao, ${ }^{15}$ J. Wang, ${ }^{15}$ J. Wang, ${ }^{15}$ X. Wang,,${ }^{15}$ Z. Wang,${ }^{15}$ H. Xiao, ${ }^{15}$ M. Xu, ${ }^{15}$ J. Zang,${ }^{15}$ Z. Zhang, ${ }^{15}$ Y. Ban ${ }^{16}$ S. Guo, ${ }^{16}$ Y. Guo, ${ }^{16}$ W. Li,${ }^{16}$ Y. Mao, ${ }^{16}$ S. J. Qian, ${ }^{16}$ H. Teng, ${ }^{16}$ B. Zhu, ${ }^{16}$ W. Zou, ${ }^{16}$ A. Cabrera, ${ }^{17}$ B. Gomez Moreno, ${ }^{17}$ A. A. Ocampo Rios, ${ }^{17}$ A. F. Osorio Oliveros, ${ }^{17}$ J. C. Sanabria, ${ }^{17}$ N. Godinovic,${ }^{18}$ D. Lelas,${ }^{18}$ K. Lelas,${ }^{18}$ R. Plestina, ${ }^{18, \mathrm{~d}}$ D. Polic,${ }^{18}$ I. Puljak, ${ }^{18}$ Z. Antunovic, ${ }^{19}$ M. Dzelalija, ${ }^{19}$ V. Brigljevic, ${ }^{20}$

S. Duric,${ }^{20}$ K. Kadija ${ }^{20}$ S. Morovic,${ }^{20}$ A. Attikis, ${ }^{21}$ M. Galanti, ${ }^{21}$ J. Mousa, ${ }^{21}$ C. Nicolaou, ${ }^{21}$ F. Ptochos,${ }^{21}$

P. A. Razis, ${ }^{21}$ M. Finger, ${ }^{22}$ M. Finger, Jr., ${ }^{22}$ Y. Assran, ${ }^{23, \mathrm{e}}$ A. Ellithi Kamel, ${ }^{23}$ S. Khalil,,${ }^{23, \mathrm{f}}$ M. A. Mahmoud, ${ }^{23, \mathrm{~g}}$ A. Hektor, ${ }^{24}$ M. Kadastik, ${ }^{24}$ M. Müntel,${ }^{24}$ M. Raidal, ${ }^{24}$ L. Rebane, ${ }^{24}$ A. Tiko, ${ }^{24}$ V. Azzolini, ${ }^{25}$ P. Eerola, ${ }^{25}$ G. Fedi, ${ }^{25}$ S. Czellar, ${ }^{26}$ J. Härkönen, ${ }^{26}$ A. Heikkinen, ${ }^{26}$ V. Karimäki, ${ }^{26}$ R. Kinnunen,${ }^{26}$ M. J. Kortelainen, ${ }^{26}$ T. Lampén, ${ }^{26}$

K. Lassila-Perini, ${ }^{26}$ S. Lehti, ${ }^{26}$ T. Lindén, ${ }^{26}$ P. Luukka, ${ }^{26}$ T. Mäenpää, ${ }^{26}$ E. Tuominen, ${ }^{26}$ J. Tuominiemi, ${ }^{26}$ E. Tuovinen, ${ }^{26}$ D. Ungaro, ${ }^{26}$ L. Wendland, ${ }^{26}$ K. Banzuzi, ${ }^{27}$ A. Karjalainen, ${ }^{27}$ A. Korpela, ${ }^{27}$ T. Tuuva, ${ }^{27}$ D. Sillou, ${ }^{28}$ M. Besancon, ${ }^{29}$ S. Choudhury, ${ }^{29}$ M. Dejardin, ${ }^{29}$ D. Denegri, ${ }^{29}$ B. Fabbro, ${ }^{29}$ J. L. Faure, ${ }^{29}$ F. Ferri, ${ }^{29}$ S. Ganjour, ${ }^{29}$ F. X. Gentit, ${ }^{29}$ A. Givernaud, ${ }^{29}$ P. Gras, ${ }^{29}$ G. Hamel de Monchenault, ${ }^{29}$ P. Jarry, ${ }^{29}$ E. Locci, ${ }^{29}$ J. Malcles, ${ }^{29}$ M. Marionneau, ${ }^{29}$ L. Millischer, ${ }^{29}$ J. Rander, ${ }^{29}$ A. Rosowsky, ${ }^{29}$ I. Shreyber, ${ }^{29}$ M. Titov, ${ }^{29}$ P. Verrecchia, ${ }^{29}$ S. Baffioni, ${ }^{30}$ F. Beaudette,${ }^{30}$ L. Benhabib, ${ }^{30}$ L. Bianchini, ${ }^{30}$ M. Bluj, ${ }^{30, h}$ C. Broutin, ${ }^{30}$ P. Busson,,${ }^{30}$ C. Charlot, ${ }^{30}$ T. Dahms,${ }^{30}$ L. Dobrzynski, ${ }^{30}$ S. Elgammal, ${ }^{30}$ R. Granier de Cassagnac, ${ }^{30}$ M. Haguenauer, ${ }^{30}$ P. Miné, ${ }^{30}$ C. Mironov, ${ }^{30}$ C. Ochando, ${ }^{30}$ P. Paganini, ${ }^{30}$ D. Sabes,${ }^{30}$ R. Salerno, ${ }^{30}$ Y. Sirois, ${ }^{30}$ C. Thiebaux,${ }^{30}$ B. Wyslouch, ${ }^{30, i}$ A. Zabi, ${ }^{30}$ J.-L. Agram, ${ }^{31, j}$ J. Andrea, ${ }^{31}$ D. Bloch, ${ }^{31}$ D. Bodin, ${ }^{31}$ J.-M. Brom,,${ }^{31}$ M. Cardaci, ${ }^{31}$ E. C. Chabert, ${ }^{31}$ C. Collard, ${ }^{31}$

E. Conte,${ }^{31, j}$ F. Drouhin, ${ }^{31, j}$ C. Ferro, ${ }^{31}$ J.-C. Fontaine, ${ }^{31, j}$ D. Gelé, ${ }^{31}$ U. Goerlach,${ }^{31}$ S. Greder, ${ }^{31}$ P. Juillot, ${ }^{31}$ M. Karim, ${ }^{31, j}$ A.-C. Le Bihan, ${ }^{31}$ Y. Mikami, ${ }^{31}$ P. Van Hove, ${ }^{31}$ F. Fassi, ${ }^{32}$ D. Mercier, ${ }^{32}$ C. Baty, ${ }^{33}$ S. Beauceron, ${ }^{33}$ N. Beaupere, ${ }^{33}$ M. Bedjidian, ${ }^{33}$ O. Bondu, ${ }^{33}$ G. Boudoul,,${ }^{33}$ D. Boumediene, ${ }^{33}$ H. Brun, ${ }^{33}$ J. Chasserat,${ }^{33}$ R. Chierici, ${ }^{33}$ D. Contardo, ${ }^{33}$ P. Depasse, ${ }^{33}$ H. El Mamouni, ${ }^{33}$ J. Fay,${ }^{33}$ S. Gascon,,${ }^{33}$ B. Ille, ${ }^{33}$ T. Kurca,,${ }^{33}$ T. Le Grand, ${ }^{33}$ M. Lethuillier, ${ }^{33}$ L. Mirabito, ${ }^{33}$ S. Perries,${ }^{33}$ V. Sordini, ${ }^{33}$ S. Tosi, ${ }^{33}$ Y. Tschudi, ${ }^{33}$ P. Verdier,${ }^{33}$ D. Lomidze, ${ }^{34}$ G. Anagnostou, ${ }^{35}$ S. Beranek, ${ }^{35}$ M. Edelhoff, ${ }^{35}$ L. Feld,${ }^{35}$ N. Heracleous,,${ }^{35}$ O. Hindrichs, ${ }^{35}$ R. Jussen, ${ }^{35}$ K. Klein, ${ }^{35}$ J. Merz, ${ }^{35}$ N. Mohr, ${ }^{35}$ A. Ostapchuk, ${ }^{35}$ A. Perieanu, ${ }^{35}$ F. Raupach,${ }^{35}$ J. Sammet, ${ }^{35}$ S. Schael, ${ }^{35}$ D. Sprenger, ${ }^{35}$ H. Weber, ${ }^{35}$ M. Weber, ${ }^{35}$ B. Wittmer, ${ }^{35}$ M. Ata, ${ }^{36}$ E. Dietz-Laursonn, ${ }^{36}$ M. Erdmann, ${ }^{36}$ T. Hebbeker, ${ }^{36}$ C. Heidemann, ${ }^{36}$ A. Hinzmann, ${ }^{36}$ K. Hoepfner, ${ }^{36}$ T. Klimkovich, ${ }^{36}$ D. Klingebiel, ${ }^{36}$ P. Kreuzer, ${ }^{36}$ D. Lanske, ${ }^{36, a}$ J. Lingemann, ${ }^{36}$ C. Magass, ${ }^{36}$ M. Merschmeyer, ${ }^{36}$ A. Meyer, ${ }^{36}$ P. Papacz, ${ }^{36}$ H. Pieta, ${ }^{36}$ H. Reithler, ${ }^{36}$ S. A. Schmitz,${ }^{36}$ L. Sonnenschein, ${ }^{36}$ J. Steggemann, ${ }^{36}$ D. Teyssier ${ }^{36}$ M. Bontenackels, ${ }^{37}$ M. Davids, ${ }^{37}$ M. Duda, ${ }^{37}$ G. Flügge,${ }^{37}$ H. Geenen, ${ }^{37}$ M. Giffels, ${ }^{37}$ W. Haj Ahmad,${ }^{37}$ D. Heydhausen, ${ }^{37}$ F. Hoehle, ${ }^{37}$ B. Kargoll, ${ }^{37}$ T. Kress, ${ }^{37}$ Y. Kuessel, ${ }^{37}$ A. Linn, ${ }^{37}$ A. Nowack, ${ }^{37}$ L. Perchalla, ${ }^{37}$ O. Pooth,${ }^{37}$ J. Rennefeld,${ }^{37}$ P. Sauerland ${ }^{37}$ A. Stahl, ${ }^{37}$ M. Thomas, ${ }^{37}$ D. Tornier, ${ }^{37}$ M. H. Zoeller, ${ }^{37}$ M. Aldaya Martin, ${ }^{38}$ W. Behrenhoff, ${ }^{38}$ U. Behrens, ${ }^{38}$ M. Bergholz, ${ }^{38, k}$ A. Bethani, ${ }^{38}$ K. Borras, ${ }^{38}$ A. Cakir, ${ }^{38}$ A. Campbell, ${ }^{38}$ E. Castro, ${ }^{38}$ D. Dammann, ${ }^{38}$ G. Eckerlin, ${ }^{38}$ D. Eckstein, ${ }^{38}$ A. Flossdorf, ${ }^{38}$ G. Flucke, ${ }^{38}$ A. Geiser ${ }^{38}$ J. Hauk, ${ }^{38}$ H. Jung, ${ }^{38, b}$ M. Kasemann, ${ }^{38}$ I. Katkov, ${ }^{38,1}$ P. Katsas, ${ }^{38}$ C. Kleinwort, ${ }^{38}$ H. Kluge, ${ }^{38}$ A. Knutsson, ${ }^{38}$ M. Krämer, ${ }^{38}$ D. Krücker, ${ }^{38}$ E. Kuznetsova ${ }^{38}$ W. Lange, ${ }^{38}$ W. Lohmann,,${ }^{38, k}$ R. Mankel, ${ }^{38}$ M. Marienfeld, ${ }^{38}$ I.-A. Melzer-Pellmann, ${ }^{38}$ A. B. Meyer, ${ }^{38}$ J. Mnich, ${ }^{38}$ A. Mussgiller, ${ }^{38}$ J. Olzem, ${ }^{38}$ A. Petrukhin, ${ }^{38}$ D. Pitzl, ${ }^{38}$ A. Raspereza, ${ }^{38}$ A. Raval, ${ }^{38}$ M. Rosin, ${ }^{38}$ R. Schmidt,${ }^{38, k}$ T. Schoerner-Sadenius, ${ }^{38}$ N. Sen, ${ }^{38}$

A. Spiridonov, ${ }^{38}$ M. Stein, ${ }^{38}$ J. Tomaszewska, ${ }^{38}$ R. Walsh ${ }^{38}$ C. Wissing,${ }^{38}$ C. Autermann,,${ }^{39}$ V. Blobel, ${ }^{39}$ S. Bobrovskyi, ${ }^{39}$ J. Draeger, ${ }^{39}$ H. Enderle, ${ }^{39}$ U. Gebbert, ${ }^{39}$ M. Görner, ${ }^{39}$ T. Hermanns, ${ }^{39}$ K. Kaschube,${ }^{39}$ G. Kaussen, ${ }^{39}$ H. Kirschenmann, ${ }^{39}$ R. Klanner, ${ }^{39}$ J. Lange, ${ }^{39}$ B. Mura, ${ }^{39}$ S. Naumann-Emme, ${ }^{39}$ F. Nowak, ${ }^{39}$ N. Pietsch, ${ }^{39}$ C. Sander, ${ }^{39}$ H. Schettler, ${ }^{39}$ P. Schleper, ${ }^{39}$ E. Schlieckau, ${ }^{39}$ M. Schröder,${ }^{39}$ T. Schum, ${ }^{39}$ H. Stadie, ${ }^{39}$ G. Steinbrück, ${ }^{39}$ J. Thomsen, ${ }^{39}$ C. Barth,${ }^{40}$ J. Bauer, ${ }^{40}$ J. Berger, ${ }^{40}$ V. Buege, ${ }^{40}$ T. Chwalek, ${ }^{40}$ W. De Boer, ${ }^{40}$ A. Dierlamm, ${ }^{40}$ G. Dirkes, ${ }^{40}$ M. Feindt, ${ }^{40}$ J. Gruschke, ${ }^{40}$ C. Hackstein, ${ }^{40}$ F. Hartmann, ${ }^{40}$ M. Heinrich,${ }^{40}$ H. Held, ${ }^{40}$ K. H. Hoffmann, ${ }^{40}$ S. Honc, ${ }^{40}$ J. R. Komaragiri, ${ }^{40}$ T. Kuhr,${ }^{40}$ D. Martschei, ${ }^{40}$ S. Mueller ${ }^{40}$ Th. Müller, ${ }^{40}$ M. Niegel, ${ }^{40}$ O. Oberst, ${ }^{40}$ A. Oehler, ${ }^{40}$ J. Ott, ${ }^{40}$ T. Peiffer, ${ }^{40}$ G. Quast, ${ }^{40}$ K. Rabbertz, ${ }^{40}$ F. Ratnikov, ${ }^{40}$ N. Ratnikova, ${ }^{40}$ M. Renz,${ }^{40}$ C. Saout, ${ }^{40}$ A. Scheurer, ${ }^{40}$ P. Schieferdecker, ${ }^{40}$ F.-P. Schilling, ${ }^{40}$ G. Schott, ${ }^{40}$ H. J. Simonis, ${ }^{40}$ F. M. Stober, ${ }^{40}$ D. Troendle, ${ }^{40}$ J. Wagner-Kuhr, ${ }^{40}$ T. Weiler ${ }^{40}$ M. Zeise, ${ }^{40}$ V. Zhukov, ${ }^{40,1}$ E. B. Ziebarth, ${ }^{40}$ G. Daskalakis,${ }^{41}$ T. Geralis, ${ }^{41}$ S. Kesisoglou, ${ }^{41}$ A. Kyriakis,${ }^{41}$ D. Loukas, ${ }^{41}$ I. Manolakos, ${ }^{41}$ A. Markou, ${ }^{41}$ C. Markou, ${ }^{41}$ C. Mavrommatis, ${ }^{41}$ E. Ntomari, ${ }^{41}$ E. Petrakou, ${ }^{41}$ L. Gouskos, ${ }^{42}$ T. J. Mertzimekis, ${ }^{42}$ A. Panagiotou, ${ }^{42}$ E. Stiliaris,${ }^{42}$ I. Evangelou, ${ }^{43}$ C. Foudas, ${ }^{43}$ P. Kokkas, ${ }^{43}$ N. Manthos, ${ }^{43}$ I. Papadopoulos, ${ }^{43}$ V. Patras, ${ }^{43}$ F. A. Triantis, ${ }^{43}$ A. Aranyi, ${ }^{44}$ 
G. Bencze,${ }^{44}$ L. Boldizsar, ${ }^{44}$ C. Hajdu, ${ }^{44, b}$ P. Hidas, ${ }^{44}$ D. Horvath, ${ }^{44, m}$ A. Kapusi,${ }^{44}$ K. Krajczar, ${ }^{44, n}$ F. Sikler, ${ }^{44, b}$ G. I. Veres ${ }^{44, n}$ G. Vesztergombi ${ }^{44, n}$ N. Beni ${ }^{45}$ J. Molnar, ${ }^{45}$ J. Palinkas, ${ }^{45}$ Z. Szillasi, ${ }^{45}$ V. Veszpremi, ${ }^{45}$ P. Raics, ${ }^{46}$ Z. L. Trocsanyi,${ }^{46}$ B. Ujvari ${ }^{46}$ S. B. Beri,${ }^{47}$ V. Bhatnagar, ${ }^{47}$ N. Dhingra,${ }^{47}$ R. Gupta, ${ }^{47}$ M. Jindal,${ }^{47}$ M. Kaur ${ }^{47}$ J. M. Kohli, ${ }^{47}$ M.Z. Mehta, ${ }^{47}$ N. Nishu, ${ }^{47}$ L. K. Saini,${ }^{47}$ A. Sharma, ${ }^{47}$ A. P. Singh,${ }^{47}$ J. Singh, ${ }^{47}$ S. P. Singh, ${ }^{47}$ S. Ahuja, ${ }^{48}$ B. C. Choudhary, ${ }^{48}$ P. Gupta, ${ }^{48}$ S. Jain, ${ }^{48}$ A. Kumar, ${ }^{48}$ A. Kumar, ${ }^{48}$ M. Naimuddin, ${ }^{48}$ K. Ranjan,${ }^{48}$ R. K. Shivpuri, ${ }^{48}$ S. Banerjee, ${ }^{49}$ S. Bhattacharya, ${ }^{49}$ S. Dutta, ${ }^{49}$ B. Gomber ${ }^{49}$ S. Jain, ${ }^{49}$ R. Khurana, ${ }^{49}$ S. Sarkar, ${ }^{49}$ R. K. Choudhury, ${ }^{50}$ D. Dutta, ${ }^{50}$ S. Kailas, ${ }^{50}$ V. Kumar, ${ }^{50}$ P. Mehta, ${ }^{50}$ A. K. Mohanty, ${ }^{50, b}$ L. M. Pant, ${ }^{50}$ P. Shukla,${ }^{50}$ T. Aziz,${ }^{51}$ M. Guchait, ${ }^{51, o}$ A. Gurtu, ${ }^{51}$ M. Maity, ${ }^{51, p}$ D. Majumder, ${ }^{51}$ G. Majumder, ${ }^{51}$ K. Mazumdar, ${ }^{51}$ G. B. Mohanty, ${ }^{51}$ A. Saha, ${ }^{51}$ K. Sudhakar, ${ }^{51}$ N. Wickramage, ${ }^{51}$ S. Banerjee, ${ }^{52}$ S. Dugad, ${ }^{52}$ N. K. Mondal,${ }^{52}$ H. Arfaei,${ }^{53}$ H. Bakhshiansohi, ${ }^{53, q}$ S. M. Etesami, ${ }^{53}$ A. Fahim,${ }^{53, q}$ M. Hashemi, ${ }^{53}$ H. Hesari, ${ }^{53}$ A. Jafari, ${ }^{53, q}$ M. Khakzad, ${ }^{53}$ A. Mohammadi, ${ }^{53, r}$ M. Mohammadi Najafabadi, ${ }^{53}$ S. Paktinat Mehdiabadi, ${ }^{53}$ B. Safarzadeh, ${ }^{53}$ M. Zeinali, ${ }^{53, \mathrm{~s}}$ M. Abbrescia, ${ }^{54 a, 54 b}$ L. Barbone, ${ }^{54 a, 54 b}$ C. Calabria, ${ }^{54 a, 54 b}$ A. Colaleo, ${ }^{54 a}$ D. Creanza, ${ }^{54 a, 54 c}$ N. De Filippis, ${ }^{54 a, 54 c, b}$ M. De Palma, ${ }^{54 a, 54 b}$ L. Fiore,${ }^{54 a}$ G. Iaselli, ${ }^{54 a, 54 c}$ L. Lusito, ${ }^{54 a, 54 b}$ G. Maggi, ${ }^{54 a, 54 c}$ M. Maggi, ${ }^{54 \mathrm{a}}$ N. Manna, ${ }^{54 \mathrm{a}, 54 \mathrm{~b}}$ B. Marangelli, ${ }^{54 \mathrm{a}, 54 \mathrm{~b}} \mathrm{~S}$. My, ${ }^{54 \mathrm{a}, 54 \mathrm{c}}$ S. Nuzzo,${ }^{54 a, 54 \mathrm{~b}}$ N. Pacifico, ${ }^{54 \mathrm{a}, 54 \mathrm{~b}}$ G. A. Pierro, ${ }^{54 \mathrm{a}}$ A. Pompili, ${ }^{54 a, 54 b}$ G. Pugliese,${ }^{54 a, 54 c}$ F. Romano, ${ }^{54 a, 54 c}$ G. Roselli, ${ }^{54 a, 54 b}$ G. Selvaggi, ${ }^{54 a, 54 b}$ L. Silvestris, ${ }^{54 a}$ R. Trentadue, ${ }^{54 \mathrm{a}}$ S. Tupputi, ${ }^{54 \mathrm{a}, 54 \mathrm{~b}}$ G. Zito, ${ }^{54 \mathrm{a}}$ G. Abbiendi, ${ }^{55 \mathrm{a}}$ A. C. Benvenuti, ${ }^{55 \mathrm{a}}$ D. Bonacorsi, ${ }^{55 \mathrm{a}}$ S. Braibant-Giacomelli, ${ }^{55 a, 55 b}$ L. Brigliadori, ${ }^{55 a}$ P. Capiluppi, ${ }^{55 a, 55 b}$ A. Castro, ${ }^{55 a, 55 b}$ F. R. Cavallo, ${ }^{55 a}$ M. Cuffiani, ${ }^{55 a, 55 b}$ G. M. Dallavalle, ${ }^{55 a}$ F. Fabbri, ${ }^{55 a}$ A. Fanfani, ${ }^{55 a, 55 b}$ D. Fasanella, ${ }^{55 a}$ P. Giacomelli, ${ }^{55 a}$ M. Giunta, ${ }^{55 a}$ C. Grandi, ${ }^{55 a}$ S. Marcellini, ${ }^{55 a}$ G. Masetti, ${ }^{55 b}$ M. Meneghelli, ${ }^{55 a, 55 b}$ A. Montanari, ${ }^{55 a}$ F. L. Navarria, ${ }^{55,55 b}$ F. Odorici, ${ }^{55 a}$ A. Perrotta, ${ }^{55 a}$ F. Primavera, ${ }^{55 a}$ A. M. Rossi, ${ }^{55 a, 55 b}$ T. Rovelli, ${ }^{55 a, 55 b}$ G. Siroli, ${ }^{55 a, 55 b}$ R. Travaglini, ${ }^{55 a, 55 b}$ S. Albergo, ${ }^{56 a, 56 b}$ G. Cappello, ${ }^{56 a, 56 b}$ M. Chiorboli, ${ }^{56 a, 56 b, b}$ S. Costa, ${ }^{56 a, 56 b}$ A. Tricomi,${ }^{56 a, 56 b}$ C. Tuve, ${ }^{56 a, 56 b}$ G. Barbagli, ${ }^{57 a}$ V. Ciulli, ${ }^{57 a, 57 b}$ C. Civinini, ${ }^{57 a}$ R. D’ Alessandro, ${ }^{57 a, 57 b}$ E. Focardi, ${ }^{57 a, 57 b}$ S. Frosali, ${ }^{57 a, 57 b}$ E. Gallo, ${ }^{57 a}$ S. Gonzi, ${ }^{57 a, 57 b}$ P. Lenzi, ${ }^{57 a, 57 b}$ M. Meschini, ${ }^{57 a}$ S. Paoletti, ${ }^{57 a}$ G. Sguazzoni, ${ }^{57 \mathrm{a}}$ A. Tropiano, ${ }^{57 \mathrm{a}, \mathrm{b}}$ L. Benussi, ${ }^{58} \mathrm{~S}$. Bianco, ${ }^{58}$ S. Colafranceschi,${ }^{58, \mathrm{t}}$ F. Fabbri, ${ }^{58}$ D. Piccolo, ${ }^{58}$ P. Fabbricatore, ${ }^{59}$ R. Musenich, ${ }^{59}$ A. Benaglia,${ }^{60 a, 60 b}$ F. De Guio, ${ }^{60 a, 60 b, b}$ L. Di Matteo, ${ }^{60 a, 60 b}$ S. Gennai, ${ }^{60 a, b}$ A. Ghezzi, ${ }^{60 a, 60 b}$ S. Malvezzi, ${ }^{60 a}$ A. Martelli, ${ }^{60 a, 60 b}$ A. Massironi, ${ }^{60 a, 60 b}$ D. Menasce, ${ }^{60 a}$ L. Moroni, ${ }^{60 a}$ M. Paganoni, ${ }^{60 a, 60 b}$ D. Pedrini, ${ }^{60 a}$ S. Ragazzi, ${ }^{60 a, 60 b}$ N. Redaelli, ${ }^{60 a}$ S. Sala,${ }^{60 a}$ T. Tabarelli de Fatis, ${ }^{60 a, 60 b}$ S. Buontempo ${ }^{61 a}$ C. A. Carrillo Montoya ${ }^{61 a, b}$ N. Cavallo, ${ }^{61 a, u}$ A. De Cosa, ${ }^{61 a, 61 b}$ F. Fabozzi, ${ }^{61 a, u}$ A. O. M. Iorio, ${ }^{61 a, b}$

L. Lista, ${ }^{61 a}$ M. Merola, ${ }^{61 a, 61 b}$ P. Paolucci, ${ }^{61 a}$ P. Azzi, ${ }^{62 a}$ N. Bacchetta, ${ }^{62 a}$ P. Bellan, ${ }^{62 a, 62 b}$ D. Bisello, ${ }^{62 a, 62 b}$

A. Branca, ${ }^{62 a}$ R. Carlin, ${ }^{62 a, 62 b}$ P. Checchia, ${ }^{62 a}$ T. Dorigo, ${ }^{62 a}$ U. Dosselli, ${ }^{62 a}$ F. Fanzago, ${ }^{62 a}$ F. Gasparini, ${ }^{62 a, 62 b}$ U. Gasparini, ${ }^{62 \mathrm{a}, 62 \mathrm{~b}}$ A. Gozzelino, ${ }^{62 \mathrm{a}}$ S. Lacaprara, ${ }^{62 \mathrm{a}}$ I. Lazzizzera, ${ }^{62 \mathrm{a}, 62 \mathrm{c}}$ M. Margoni, ${ }^{62 \mathrm{a}, 62 \mathrm{~b}} \mathrm{M}$. Mazzucato, ${ }^{62 \mathrm{a}}$ A. T. Meneguzzo, ${ }^{62 a, 62 b}$ M. Nespolo, ${ }^{62 a, b}$ L. Perrozzi,${ }^{62 a, b}$ N. Pozzobon, ${ }^{62 a, 62 b}$ P. Ronchese, ${ }^{62 a, 62 b}$ F. Simonetto, ${ }^{62 a, 62 b}$ E. Torassa, ${ }^{62 \mathrm{a}}$ M. Tosi, ${ }^{62 \mathrm{a}, 62 \mathrm{~b}}$ S. Vanini, ${ }^{62 \mathrm{a}, 62 \mathrm{~b}}$ P. Zotto, ${ }^{62 \mathrm{a}, 62 \mathrm{~b}}$ G. Zumerle, ${ }^{62 \mathrm{a}, 62 \mathrm{~b}}$ P. Baesso, ${ }^{63 \mathrm{a}, 63 \mathrm{~b}}$ U. Berzano, ${ }^{63 \mathrm{a}}$ S. P. Ratti, ${ }^{63 a, 63 b}$ C. Riccardi, ${ }^{63 a, 63 b}$ P. Torre, ${ }^{63 a, 63 b}$ P. Vitulo, ${ }^{63 a, 63 b}$ C. Viviani, ${ }^{63 a, 63 b}$ M. Biasini, ${ }^{64 a, 64 b}$ G. M. Bilei, ${ }^{64 a}$

B. Caponeri, ${ }^{64 a, 64 b}$ L. Fanò, ${ }^{64 a, 64 b}$ P. Lariccia, ${ }^{64 a, 64 b}$ A. Lucaroni, ${ }^{64 a, 64 b, b}$ G. Mantovani, ${ }^{64 a, 64 b}$ M. Menichelli, ${ }^{64 a}$

A. Nappi ${ }^{64 a, 64 b}$ F. Romeo, ${ }^{64 a, 64 b}$ A. Santocchia,${ }^{64 a, 64 b}$ S. Taroni, ${ }^{64 a, 64 b, b}$ M. Valdata, ${ }^{64 a, 64 b}$ P. Azzurri, ${ }^{65 a, 65 c}$

G. Bagliesi, ${ }^{65 a}$ J. Bernardini, ${ }^{65 a, 65 b}$ T. Boccali, ${ }^{65 a, b}$ G. Broccolo, ${ }^{65 a, 65 c}$ R. Castaldi, ${ }^{65 a}$ R. T. D’ Agnolo, ${ }^{65 a, 65 c}$

R. Dell'Orso, ${ }^{65 a}$ F. Fiori, ${ }^{65 a, 65 b}$ L. Foà, ${ }^{65 a, 65 c}$ A. Giassi, ${ }^{65 a}$ A. Kraan, ${ }^{65 a}$ F. Ligabue,${ }^{65 a, 65 c}$ T. Lomtadze, ${ }^{65 a}$

L. Martini, ${ }^{65 a, v}$ A. Messineo, ${ }^{65 a, 65 b}$ F. Palla, ${ }^{65 a}$ G. Segneri, ${ }^{65 a}$ A. T. Serban ${ }^{65 a}$ P. Spagnolo, ${ }^{65 a}$ R. Tenchini, ${ }^{65 a}$

G. Tonelli, ${ }^{65 a, 65 b, b}$ A. Venturi, ${ }^{65 a, b}$ P. G. Verdini, ${ }^{65 a}$ L. Barone, ${ }^{66 a, 66 b}$ F. Cavallari, ${ }^{66 a}$ D. Del Re,${ }^{66 a, 66 b}$

E. Di Marco ${ }^{66 a, 66 b}$ M. Diemoz ${ }^{66 a}$ D. Franci, ${ }^{66 a, 66 b}$ M. Grassi, ${ }^{66 a, b}$ E. Longo,${ }^{66 a, 66 b}$ P. Meridiani, ${ }^{66 a}$ S. Nourbakhsh, ${ }^{66 a}$ G. Organtini, ${ }^{66 a, 66 b}$ F. Pandolfi, ${ }^{66 a, 66 b, b}$ R. Paramatti, ${ }^{66 a}$ S. Rahatlou, ${ }^{66 a, 66 b}$ C. Rovelli, ${ }^{66 a, b}$ N. Amapane, ${ }^{67 a, 67 b}$ R. Arcidiacono, ${ }^{67 a, 67 \mathrm{c}}$ S. Argiro, ${ }^{67 a, 67 b}$ M. Arneodo, ${ }^{67 a, 67 \mathrm{c}}$ C. Biino, ${ }^{67 \mathrm{a}}$ C. Botta, ${ }^{67 a, 67 \mathrm{~b}, \mathrm{~b}}$ N. Cartiglia, ${ }^{67 a}$ R. Castello, ${ }^{67 a, 67 b}$ M. Costa, ${ }^{67 a, 67 b}$ N. Demaria, ${ }^{67 a}$ A. Graziano, ${ }^{67 a, 67 b, b}$ C. Mariotti, ${ }^{67 a}$ M. Marone, ${ }^{67 a, 67 b}$ S. Maselli, ${ }^{67 a}$ E. Migliore, ${ }^{67 a, 67 b}$ G. Mila, ${ }^{67 a, 67 b}$ V. Monaco, ${ }^{67 a, 67 b}$ M. Musich, ${ }^{67 a, 67 b}$ M. M. Obertino,${ }^{67 a, 67 \mathrm{c}}$ N. Pastrone, ${ }^{67 \mathrm{a}}$ M. Pelliccioni, ${ }^{67 \mathrm{a}, 67 \mathrm{~b}}$ A. Potenza, ${ }^{67 \mathrm{a}, 67 \mathrm{~b}}$ A. Romero, ${ }^{67 \mathrm{a}, 67 \mathrm{~b}}$ M. Ruspa, ${ }^{67 \mathrm{a}, 67 \mathrm{c}}$ R. Sacchi, ${ }^{67 a, 67 b}$ V. Sola, ${ }^{67 a, 67 b}$ A. Solano, ${ }^{67 a, 67 b}$ A. Staiano, ${ }^{67 a}$ A. Vilela Pereira, ${ }^{67 a}$ S. Belforte, ${ }^{68 a}$ F. Cossutti, ${ }^{68 a}$ G. Della Ricca, ${ }^{68 a, 68 b}$ B. Gobbo, ${ }^{68 a}$ D. Montanino, ${ }^{68 a, 68 b}$ A. Penzo, ${ }^{68 a}$ S. G. Heo, ${ }^{69}$ S. K. Nam, ${ }^{69}$ S. Chang, ${ }^{70}$ J. Chung, ${ }^{70}$ D. H. Kim, ${ }^{70}$ G. N. Kim, ${ }^{70}$ J.E. Kim, ${ }^{70}$ D. J. Kong, ${ }^{70}$ H. Park, ${ }^{70}$ S. R. Ro, ${ }^{70}$ D. C. Son, ${ }^{70}$ T. Son, ${ }^{70}$ Zero Kim, ${ }^{71}$ J. Y. Kim, ${ }^{71}$ S. Song, ${ }^{71}$ S. Choi, ${ }^{72}$ B. Hong, ${ }^{72}$ M. Jo, ${ }^{72}$ H. Kim ${ }^{72}$ J. H. Kim, ${ }^{72}$ T. J. Kim, ${ }^{72}$ K. S. Lee, ${ }^{72}$ D. H. Moon, ${ }^{72}$ S. K. Park, ${ }^{72}$ K. S. Sim,${ }^{72}$ M. Choi,${ }^{73}$ S. Kang, ${ }^{73}$ H. Kim, ${ }^{73}$ C. Park,${ }^{73}$ I. C. Park, ${ }^{73}$ S. Park,${ }^{73}$ G. Ryu, ${ }^{73}$ 
Y. Choi, ${ }^{74}$ Y. K. Choi, ${ }^{74}$ J. Goh, ${ }^{74}$ M. S. Kim,${ }^{74}$ J. Lee, ${ }^{74}$ S. Lee, ${ }^{74}$ H. Seo, ${ }^{74}$ I. Yu,${ }^{74}$ M. J. Bilinskas,${ }^{75}$ I. Grigelionis,${ }^{75}$ M. Janulis, ${ }^{75}$ D. Martisiute, ${ }^{75}$ P. Petrov, ${ }^{75}$ T. Sabonis, ${ }^{75}$ H. Castilla-Valdez,${ }^{76}$ E. De La Cruz-Burelo, ${ }^{76}$

I. Heredia-de La Cruz, ${ }^{76}$ R. Lopez-Fernandez,${ }^{76}$ R. Magaña Villalba, ${ }^{76}$ A. Sánchez-Hernández, ${ }^{76}$

L. M. Villasenor-Cendejas, ${ }^{76}$ S. Carrillo Moreno, ${ }^{77}$ F. Vazquez Valencia ${ }^{77}$ H. A. Salazar Ibarguen, ${ }^{78}$

E. Casimiro Linares, ${ }^{79}$ A. Morelos Pineda, ${ }^{79}$ M. A. Reyes-Santos, ${ }^{79}$ D. Krofcheck, ${ }^{80}$ J. Tam,${ }^{80}$ P. H. Butler, ${ }^{81}$

R. Doesburg, ${ }^{81}$ H. Silverwood, ${ }^{81}$ M. Ahmad, ${ }^{82}$ I. Ahmed, ${ }^{82}$ M. I. Asghar, ${ }^{82}$ H. R. Hoorani, ${ }^{82}$ W. A. Khan, ${ }^{82}$

T. Khurshid, ${ }^{82}$ S. Qazi, ${ }^{82}$ G. Brona, ${ }^{83}$ M. Cwiok, ${ }^{83}$ W. Dominik, ${ }^{83}$ K. Doroba, ${ }^{83}$ A. Kalinowski, ${ }^{83}$ M. Konecki, ${ }^{83}$ J. Krolikowski, ${ }^{83}$ T. Frueboes ${ }^{84}$ R. Gokieli, ${ }^{84}$ M. Górski, ${ }^{84}$ M. Kazana, ${ }^{84}$ K. Nawrocki, ${ }^{84}$

K. Romanowska-Rybinska, ${ }^{84}$ M. Szleper, ${ }^{84}$ G. Wrochna,${ }^{84}$ P. Zalewski, ${ }^{84}$ N. Almeida ${ }^{85}$ P. Bargassa, ${ }^{85}$ A. David ${ }^{85}$

P. Faccioli, ${ }^{85}$ P. G. Ferreira Parracho, ${ }^{85}$ M. Gallinaro, ${ }^{85, b}$ P. Musella, ${ }^{85}$ A. Nayak, ${ }^{85}$ J. Pela, ${ }^{85, b}$ P. Q. Ribeiro, ${ }^{85}$

J. Seixas, ${ }^{85}$ J. Varela ${ }^{85}$ S. Afanasiev,${ }^{86}$ I. Belotelov ${ }^{86}$ P. Bunin,${ }^{86}$ I. Golutvin ${ }^{86}$ A. Kamenev, ${ }^{86}$ V. Karjavin, ${ }^{86}$

G. Kozlov, ${ }^{86}$ A. Lanev, ${ }^{86}$ P. Moisenz,${ }^{86}$ V. Palichik, ${ }^{86}$ V. Perelygin, ${ }^{86}$ S. Shmatov, ${ }^{86}$ V. Smirnov, ${ }^{86}$ A. Volodko, ${ }^{86}$

A. Zarubin, ${ }^{86}$ V. Golovtsov, ${ }^{87}$ Y. Ivanov, ${ }^{87}$ V. Kim, ${ }^{87}$ P. Levchenko, ${ }^{87}$ V. Murzin, ${ }^{87}$ V. Oreshkin,${ }^{87}$ I. Smirnov, ${ }^{87}$

V. Sulimov, ${ }^{87}$ L. Uvarov, ${ }^{87}$ S. Vavilov, ${ }^{87}$ A. Vorobyev, ${ }^{87}$ An. Vorobyev,${ }^{87}$ Yu. Andreev, ${ }^{88}$ A. Dermenev, ${ }^{88}$

S. Gninenko, ${ }^{88}$ N. Golubev, ${ }^{88}$ M. Kirsanov, ${ }^{88}$ N. Krasnikov, ${ }^{88}$ V. Matveev, ${ }^{88}$ A. Pashenkov, ${ }^{88}$ A. Toropin,${ }^{88}$

S. Troitsky, ${ }^{88}$ V. Epshteyn, ${ }^{89}$ V. Gavrilov,${ }^{89}$ V. Kaftanov, ${ }^{89, a}$ M. Kossov, ${ }^{89, b}$ A. Krokhotin, ${ }^{89}$ N. Lychkovskaya, ${ }^{89}$

V. Popov, ${ }^{89}$ G. Safronov, ${ }^{89}$ S. Semenov, ${ }^{89}$ V. Stolin, ${ }^{89}$ E. Vlasov, ${ }^{89}$ A. Zhokin, ${ }^{89}$ E. Boos, ${ }^{90}$ A. Ershov, ${ }^{90}$

A. Gribushin, ${ }^{90}$ O. Kodolova, ${ }^{90}$ V. Korotkikh, ${ }^{90}$ I. Lokhtin, ${ }^{90}$ A. Markina, ${ }^{90}$ S. Obraztsov, ${ }^{90}$ M. Perfilov, ${ }^{90}$

S. Petrushanko, ${ }^{90}$ L. Sarycheva, ${ }^{90}$ V. Savrin,,${ }^{90}$ A. Snigirev, ${ }^{90}$ I. Vardanyan,,${ }^{90}$ V. Andreev, ${ }^{91}$ M. Azarkin, ${ }^{91}$

I. Dremin, ${ }^{91}$ M. Kirakosyan,${ }^{91}$ A. Leonidov, ${ }^{91}$ S. V. Rusakov, ${ }^{91}$ A. Vinogradov, ${ }^{91}$ I. Azhgirey,${ }^{92}$ I. Bayshev, ${ }^{92}$

S. Bitioukov, ${ }^{92}$ V. Grishin, ${ }^{92, b}$ V. Kachanov,${ }^{92}$ D. Konstantinov, ${ }^{92}$ A. Korablev, ${ }^{92}$ V. Krychkine,${ }^{92}$ V. Petrov, ${ }^{92}$

R. Ryutin, ${ }^{92}$ A. Sobol, ${ }^{92}$ L. Tourtchanovitch, ${ }^{92}$ S. Troshin, ${ }^{92}$ N. Tyurin, ${ }^{92}$ A. Uzunian, ${ }^{92}$ A. Volkov, ${ }^{92}$ P. Adzic,,${ }^{93, w}$ M. Djordjevic, ${ }^{93}$ D. Krpic, ${ }^{93, w}$ J. Milosevic, ${ }^{93}$ M. Aguilar-Benitez, ${ }^{94}$ J. Alcaraz Maestre,,${ }^{94}$ P. Arce,${ }^{94}$ C. Battilana, ${ }^{94}$ E. Calvo, ${ }^{94}$ M. Cepeda, ${ }^{94}$ M. Cerrada,${ }^{94}$ M. Chamizo Llatas, ${ }^{94}$ N. Colino, ${ }^{94}$ B. De La Cruz,${ }^{94}$ A. Delgado Peris, ${ }^{94}$

C. Diez Pardos, ${ }^{94}$ D. Domínguez Vázquez, ${ }^{94}$ C. Fernandez Bedoya,${ }^{94}$ J. P. Fernández Ramos, ${ }^{94}$ A. Ferrando, ${ }^{94}$

J. Flix, ${ }^{94}$ M. C. Fouz, ${ }^{94}$ P. Garcia-Abia, ${ }^{94}$ O. Gonzalez Lopez,${ }^{94}$ S. Goy Lopez,${ }^{94}$ J. M. Hernandez, ${ }^{94}$ M. I. Josa, ${ }^{94}$

G. Merino, ${ }^{94}$ J. Puerta Pelayo, ${ }^{94}$ I. Redondo,${ }^{94}$ L. Romero, ${ }^{94}$ J. Santaolalla, ${ }^{94}$ M. S. Soares,${ }^{94}$ C. Willmott,${ }^{94}$

C. Albajar, ${ }^{95}$ G. Codispoti, ${ }^{95}$ J. F. de Trocóniz, ${ }^{95}$ J. Cuevas, ${ }^{96}$ J. Fernandez Menendez, ${ }^{96}$ S. Folgueras, ${ }^{96}$

I. Gonzalez Caballero, ${ }^{96}$ L. Lloret Iglesias, ${ }^{96}$ J. M. Vizan Garcia, ${ }^{96}$ J. A. Brochero Cifuentes, ${ }^{97}$ I. J. Cabrillo, ${ }^{97}$

A. Calderon, ${ }^{97}$ S. H. Chuang, ${ }^{97}$ J. Duarte Campderros, ${ }^{97}$ M. Felcini,,${ }^{97, x}$ M. Fernandez,${ }^{97}$ G. Gomez,${ }^{97}$

J. Gonzalez Sanchez, ${ }^{97}$ C. Jorda, ${ }^{97}$ P. Lobelle Pardo, ${ }^{97}$ A. Lopez Virto, ${ }^{97}$ J. Marco, ${ }^{97}$ R. Marco, ${ }^{97}$

C. Martinez Rivero, ${ }^{97}$ F. Matorras, ${ }^{97}$ F. J. Munoz Sanchez, ${ }^{97}$ J. Piedra Gomez, ${ }^{97, y}$ T. Rodrigo, ${ }^{97}$

A. Y. Rodríguez-Marrero, ${ }^{97}$ A. Ruiz-Jimeno, ${ }^{97}$ L. Scodellaro, ${ }^{97}$ M. Sobron Sanudo, ${ }^{97}$ I. Vila,,${ }^{97}$

R. Vilar Cortabitarte, ${ }^{97}$ D. Abbaneo, ${ }^{98}$ E. Auffray, ${ }^{98}$ G. Auzinger,${ }^{98}$ P. Baillon, ${ }^{98}$ A. H. Ball, ${ }^{98}$ D. Barney,${ }^{98}$

A. J. Bell, ${ }^{98, z}$ D. Benedetti, ${ }^{98}$ C. Bernet,${ }^{98, d}$ W. Bialas, ${ }^{98}$ P. Bloch, ${ }^{98}$ A. Bocci, ${ }^{98}$ S. Bolognesi,${ }^{98}$ M. Bona,${ }^{98}$

H. Breuker, ${ }^{98}$ K. Bunkowski, ${ }^{98}$ T. Camporesi, ${ }^{98}$ G. Cerminara, ${ }^{98}$ J. A. Coarasa Perez,${ }^{98}$ B. Curé, ${ }^{98}$ D. D'Enterria, ${ }^{98}$

A. De Roeck, ${ }^{98}$ S. Di Guida, ${ }^{98}$ N. Dupont-Sagorin, ${ }^{98}$ A. Elliott-Peisert, ${ }^{98}$ B. Frisch, ${ }^{98}$ W. Funk,${ }^{98}$ A. Gaddi,${ }^{98}$

G. Georgiou, ${ }^{98}$ H. Gerwig, ${ }^{98}$ D. Gigi, ${ }^{98}$ K. Gill, ${ }^{98}$ D. Giordano, ${ }^{98}$ F. Glege, ${ }^{98}$ R. Gomez-Reino Garrido, ${ }^{98}$

M. Gouzevitch, ${ }^{98}$ P. Govoni, ${ }^{98}$ S. Gowdy, ${ }^{98}$ L. Guiducci, ${ }^{98}$ M. Hansen, ${ }^{98}$ C. Hartl,${ }^{98}$ J. Harvey, ${ }^{98}$ J. Hegeman,${ }^{98}$ B. Hegner, ${ }^{98}$ H. F. Hoffmann, ${ }^{98}$ A. Honma, ${ }^{98}$ V. Innocente, ${ }^{98}$ P. Janot,${ }^{98}$ K. Kaadze, ${ }^{98}$ E. Karavakis, ${ }^{98}$ P. Lecoq,${ }^{98}$ C. Lourenço, ${ }^{98}$ T. Mäki, ${ }^{98}$ M. Malberti, ${ }^{98}$ L. Malgeri,${ }^{98}$ M. Mannelli, ${ }^{98}$ L. Masetti,${ }^{98}$ A. Maurisset,${ }^{98}$ F. Meijers,${ }^{98}$ S. Mersi ${ }^{98}$ E. Meschi, ${ }^{98}$ R. Moser, ${ }^{98}$ M. U. Mozer,${ }^{98}$ M. Mulders, ${ }^{98}$ E. Nesvold, ${ }^{98, b}$ M. Nguyen,${ }^{98}$ T. Orimoto, ${ }^{98}$ L. Orsini, ${ }^{98}$ E. Palencia Cortezon, ${ }^{98}$ E. Perez,${ }^{98}$ A. Petrilli, ${ }^{98}$ A. Pfeiffer, ${ }^{98}$ M. Pierini, ${ }^{98}$ M. Pimiä, ${ }^{98}$ D. Piparo, ${ }^{98}$ G. Polese, ${ }^{98}$ A. Racz, ${ }^{98}$ W. Reece, ${ }^{98}$ J. Rodrigues Antunes, ${ }^{98}$ G. Rolandi, ${ }^{98, a a}$ T. Rommerskirchen, ${ }^{98}$ M. Rovere, ${ }^{98}$ H. Sakulin, ${ }^{98}$ C. Schäfer, ${ }^{98}$ C. Schwick, ${ }^{98}$ I. Segoni,${ }^{98}$ A. Sharma, ${ }^{98}$ P. Siegrist,${ }^{98}$ M. Simon, ${ }^{98}$ P. Sphicas, ${ }^{98, b b}$ M. Spiropulu, ${ }^{98, c c}$ M. Stoye,${ }^{98}$ P. Tropea, ${ }^{98}$ A. Tsirou,,${ }^{98}$ P. Vichoudis,,${ }^{98}$ M. Voutilainen, ${ }^{98}$ W. D. Zeuner,${ }^{98}$ W. Bertl,${ }^{99}$ K. Deiters, ${ }^{99}$ W. Erdmann, ${ }^{99}$ K. Gabathuler, ${ }^{99}$ R. Horisberger,${ }^{99}$ Q. Ingram, ${ }^{99}$ H. C. Kaestli, ${ }^{99}$ S. König, ${ }^{99}$

D. Kotlinski, ${ }^{99}$ U. Langenegger, ${ }^{99}$ F. Meier, ${ }^{99}$ D. Renker, ${ }^{99}$ T. Rohe, ${ }^{99}$ J. Sibille,${ }^{99, d d}$ A. Starodumov, ${ }^{99, \text { ee }}$ L. Bäni, ${ }^{100}$

P. Bortignon, ${ }^{100}$ L. Caminada, ${ }^{100, f f}$ B. Casal, ${ }^{100}$ N. Chanon, ${ }^{100}$ Z. Chen, ${ }^{100}$ S. Cittolin, ${ }^{100}$ G. Dissertori,,${ }^{100}$

M. Dittmar, ${ }^{100}$ J. Eugster, ${ }^{100}$ K. Freudenreich, ${ }^{100}$ C. Grab, ${ }^{100}$ W. Hintz, ${ }^{100}$ P. Lecomte, ${ }^{100}$ W. Lustermann, ${ }^{100}$ C. Marchica, ${ }^{100, \text { ff }}$ P. Martinez Ruiz del Arbol, ${ }^{100}$ P. Milenovic, ${ }^{100, g g}$ F. Moortgat, ${ }^{100}$ C. Nägeli, ${ }^{100, f f}$ P. Nef, ${ }^{100}$ 
F. Nessi-Tedaldi, ${ }^{100}$ L. Pape,${ }^{100}$ F. Pauss, ${ }^{100}$ T. Punz, ${ }^{100}$ A. Rizzi, ${ }^{100}$ F. J. Ronga, ${ }^{100}$ M. Rossini, ${ }^{100}$ L. Sala, ${ }^{100}$ A. K. Sanchez, ${ }^{100}$ M.-C. Sawley, ${ }^{100}$ B. Stieger, ${ }^{100}$ L. Tauscher, ${ }^{100, a}$ A. Thea, ${ }^{100}$ K. Theofilatos, ${ }^{100}$ D. Treille, ${ }^{100}$ C. Urscheler, ${ }^{100}$ R. Wallny, ${ }^{100}$ M. Weber, ${ }^{100}$ L. Wehrli, ${ }^{100}$ J. Weng, ${ }^{100}$ E. Aguilo, ${ }^{101}$ C. Amsler, ${ }^{101}$ V. Chiochia, ${ }^{101}$ S. De Visscher, ${ }^{101}$ C. Favaro, ${ }^{101}$ M. Ivova Rikova, ${ }^{101}$ B. Millan Mejias, ${ }^{101}$ P. Otiougova, ${ }^{101}$ P. Robmann, ${ }^{101}$ A. Schmidt, ${ }^{101}$ H. Snoek, ${ }^{101}$ Y. H. Chang, ${ }^{102}$ K. H. Chen, ${ }^{102}$ C. M. Kuo, ${ }^{102}$ S. W. Li, ${ }^{102}$ W. Lin, ${ }^{102}$ Z. K. Liu, ${ }^{102}$ Y. J. Lu, ${ }^{102}$ D. Mekterovic, ${ }^{102}$ R. Volpe, ${ }^{102}$ J. H. Wu, ${ }^{102}$ S. S. Yu, ${ }^{102}$ P. Bartalini, ${ }^{103}$ P. Chang, ${ }^{103}$ Y. H. Chang, ${ }^{103}$ Y. W. Chang, ${ }^{103}$ Y. Chao, ${ }^{103}$ K. F. Chen, ${ }^{103}$ W.-S. Hou, ${ }^{103}$ Y. Hsiung, ${ }^{103}$ K. Y. Kao, ${ }^{103}$ Y. J. Lei, ${ }^{103}$ R.-S. Lu, ${ }^{103}$ J. G. Shiu, ${ }^{103}$ Y. M. Tzeng, ${ }^{103}$ M. Wang, ${ }^{103}$ A. Adiguzel, ${ }^{104}$ M. N. Bakirci, ${ }^{104, h h}$ S. Cerci, ${ }^{104, \text { ii }}$ C. Dozen, ${ }^{104}$ I. Dumanoglu, ${ }^{104}$ E. Eskut, ${ }^{104}$ S. Girgis, ${ }^{104}$ G. Gokbulut, ${ }^{104}$ I. Hos, ${ }^{104}$ E. E. Kangal, ${ }^{104}$ A. Kayis Topaksu, ${ }^{104}$ G. Onengut, ${ }^{104}$ K. Ozdemir, ${ }^{104}$ S. Ozturk, ${ }^{104, j j}$ A. Polatoz, ${ }^{104}$ K. Sogut, ${ }^{104, k k}$ D. Sunar Cerci, ${ }^{104, i i}$ B. Tali, ${ }^{104, i i}$ H. Topakli, ${ }^{104, \text { hh }}$ D. Uzun, ${ }^{104}$ L. N. Vergili, ${ }^{104}$ M. Vergili, ${ }^{104}$ I. V. Akin, ${ }^{105}$ T. Aliev, ${ }^{105}$ B. Bilin, ${ }^{105}$ S. Bilmis, ${ }^{105}$ M. Deniz, ${ }^{105}$ H. Gamsizkan, ${ }^{105}$ A. M. Guler, ${ }^{105}$ K. Ocalan, ${ }^{105}$ A. Ozpineci, ${ }^{105}$ M. Serin, ${ }^{105}$ R. Sever, ${ }^{105}$ U. E. Surat, ${ }^{105}$ E. Yildirim, ${ }^{105}$ M. Zeyrek, ${ }^{105}$ M. Deliomeroglu, ${ }^{106}$ D. Demir, ${ }^{106,11}$ E. Gülmez, ${ }^{106}$ B. Isildak, ${ }^{106}$ M. Kaya, ${ }^{106, m m}$ O. Kaya, ${ }^{106, m m}$ M. Özbek, ${ }^{106}$ S. Ozkorucuklu, ${ }^{106, n n}$ N. Sonmez, ${ }^{106, \text { oo }}{ }^{\text {L. Levchuk, }}{ }^{107}$ F. Bostock, ${ }^{108}$ J. J. Brooke, ${ }^{108}$ T. L. Cheng, ${ }^{108}$ E. Clement, ${ }^{108}$ D. Cussans, ${ }^{108}$ R. Frazier, ${ }^{108}$ J. Goldstein, ${ }^{108}$ M. Grimes, ${ }^{108}$ D. Hartley, ${ }^{108}$ G. P. Heath, ${ }^{108}$ H. F. Heath, ${ }^{108}$ L. Kreczko, ${ }^{108}$ S. Metson, ${ }^{108}$ D. M. Newbold, ${ }^{108, p p}$ K. Nirunpong, ${ }^{108}$ A. Poll, ${ }^{108}$ S. Senkin, ${ }^{108}$ V. J. Smith, ${ }^{108}$ L. Basso, ${ }^{109, q q}$ A. Belyaev, ${ }^{109, q q}$ C. Brew, ${ }^{109}$ R. M. Brown, ${ }^{109}$ B. Camanzi, ${ }^{109}$ D. J. A. Cockerill, ${ }^{109}$ J. A. Coughlan, ${ }^{109}$ K. Harder, ${ }^{109}$ S. Harper, ${ }^{109}$ J. Jackson, ${ }^{109}$ B. W. Kennedy, ${ }^{109}$ E. Olaiya, ${ }^{109}$ D. Petyt, ${ }^{109}$ B. C. Radburn-Smith, ${ }^{109}$ C. H. Shepherd-Themistocleous, ${ }^{109}$ I. R. Tomalin, ${ }^{109}$ W. J. Womersley, ${ }^{109}$ S. D. Worm,${ }^{109}$ R. Bainbridge,${ }^{110}$ G. Ball, ${ }^{110}$ J. Ballin, ${ }^{110}$ R. Beuselinck, ${ }^{110}$ O. Buchmuller, ${ }^{110}$ D. Colling, ${ }^{110}$ N. Cripps, ${ }^{110}$ M. Cutajar, ${ }^{10}$ G. Davies, ${ }^{110}$ M. Della Negra, ${ }^{110}$ W. Ferguson, ${ }^{10}$ J. Fulcher, ${ }^{110}$ D. Futyan, ${ }^{110}$ A. Gilbert, ${ }^{110}$ A. Guneratne Bryer, ${ }^{110}$ G. Hall, ${ }^{110}$ Z. Hatherell, ${ }^{110}$ J. Hays, ${ }^{110}$ G. Iles, ${ }^{110}$ M. Jarvis, ${ }^{110}$ G. Karapostoli, ${ }^{110}$ L. Lyons, ${ }^{110}$ B. C. MacEvoy, ${ }^{110}$ A.-M. Magnan, ${ }^{110}$ J. Marrouche, ${ }^{110}$ B. Mathias, ${ }^{110}$ R. Nandi, ${ }^{110}$ J. Nash, ${ }^{110}$ A. Nikitenko, ${ }^{110, e e}$ A. Papageorgiou, ${ }^{110}$ M. Pesaresi, ${ }^{110}$ K. Petridis, ${ }^{110}$ M. Pioppi, ${ }^{110, r r}$ D. M. Raymond, ${ }^{110}$ S. Rogerson, ${ }^{110}$ N. Rompotis, ${ }^{110}$ A. Rose, ${ }^{110}$ M. J. Ryan, ${ }^{110}$ C. Seez, ${ }^{110}$ P. Sharp, ${ }^{110}$ A. Sparrow, ${ }^{110}$ A. Tapper, ${ }^{110}$ S. Tourneur, ${ }^{110}$ M. Vazquez Acosta, ${ }^{110}$ T. Virdee, ${ }^{110}$ S. Wakefield, ${ }^{110}$ N. Wardle, ${ }^{110}$ D. Wardrope, ${ }^{110}$ T. Whyntie, ${ }^{110}$ M. Barrett, ${ }^{111}$ M. Chadwick, ${ }^{111}$ J. E. Cole, ${ }^{111}$ P. R. Hobson, ${ }^{111}$ A. Khan, ${ }^{111}$ P. Kyberd, ${ }^{111}$ D. Leslie, ${ }^{111}$ W. Martin, ${ }^{111}$ I. D. Reid, ${ }^{111}$ L. Teodorescu, ${ }^{111}$ K. Hatakeyama, ${ }^{112}$ H. Liu, ${ }^{112}$ C. Henderson, ${ }^{113}$ T. Bose, ${ }^{114}$ E. Carrera Jarrin, ${ }^{114}$ C. Fantasia, ${ }^{114}$ A. Heister, ${ }^{114}$ J. St. John, ${ }^{114}$ P. Lawson, ${ }^{114}$ D. Lazic, ${ }^{114}$ J. Rohlf, ${ }^{114}$ D. Sperka, ${ }^{114}$ L. Sulak, ${ }^{114}$ A. Avetisyan, ${ }^{115}$ S. Bhattacharya, ${ }^{115}$ J. P. Chou, ${ }^{115}$ D. Cutts, ${ }^{115}$ A. Ferapontov, ${ }^{115}$ U. Heintz, ${ }^{115}$ S. Jabeen, ${ }^{115}$ G. Kukartsev, ${ }^{115}$ G. Landsberg, ${ }^{115}$ M. Luk, ${ }^{115}$ M. Narain, ${ }^{115}$ D. Nguyen, ${ }^{115}$ M. Segala, ${ }^{115}$ T. Sinthuprasith, ${ }^{115}$ T. Speer, ${ }^{115}$ K. V. Tsang, ${ }^{115}$ R. Breedon, ${ }^{116}$ G. Breto, ${ }^{116}$ M. Calderon De La Barca Sanchez, ${ }^{116}$ S. Chauhan, ${ }^{116}$ M. Chertok, ${ }^{116}$ J. Conway, ${ }^{116}$ P. T. Cox, ${ }^{116}$ J. Dolen, ${ }^{116}$ R. Erbacher, ${ }^{116}$ E. Friis, ${ }^{116}$ W. Ko, ${ }^{116}$ A. Kopecky, ${ }^{116}$ R. Lander, ${ }^{116}$ H. Liu, ${ }^{116}$ S. Maruyama, ${ }^{116}$ T. Miceli, ${ }^{116}$ M. Nikolic, ${ }^{116}$ D. Pellett, ${ }^{116}$ J. Robles, ${ }^{116}$ S. Salur, ${ }^{116}$ T. Schwarz, ${ }^{116}$ M. Searle, ${ }^{116}$ J. Smith, ${ }^{116}$ M. Squires, ${ }^{116}$ M. Tripathi, ${ }^{116}$ R. Vasquez Sierra, ${ }^{116}$ C. Veelken, ${ }^{116}$ V. Andreev, ${ }^{117}$ K. Arisaka, ${ }^{117}$ D. Cline, ${ }^{117}$ R. Cousins, ${ }^{117}$ A. Deisher, ${ }^{117}$ J. Duris, ${ }^{117}$ S. Erhan, ${ }^{117}$ C. Farrell, ${ }^{117}$ J. Hauser, ${ }^{117}$ M. Ignatenko, ${ }^{117}$ C. Jarvis, ${ }^{117}$ C. Plager, ${ }^{117}$ G. Rakness, ${ }^{117}$ P. Schlein, ${ }^{117, a}$ J. Tucker, ${ }^{117}$ V. Valuev, ${ }^{117}$ J. Babb, ${ }^{118}$ A. Chandra, ${ }^{118}$ R. Clare, ${ }^{118}$ J. Ellison, ${ }^{118}$ J. W. Gary, ${ }^{118}$ F. Giordano, ${ }^{118}$ G. Hanson, ${ }^{118}$ G. Y. Jeng, ${ }^{118}$ S. C. Kao, ${ }^{118}$ F. Liu, ${ }^{118}$ H. Liu, ${ }^{118}$ O. R. Long, ${ }^{118}$ A. Luthra, ${ }^{118}$ H. Nguyen, ${ }^{118}$ B. C. Shen, ${ }^{118, a}$ R. Stringer, ${ }^{118}$ J. Sturdy, ${ }^{118}$ S. Sumowidagdo, ${ }^{118}$ R. Wilken, ${ }^{118}$ S. Wimpenny, ${ }^{118}$ W. Andrews, ${ }^{119}$ J. G. Branson, ${ }^{119}$ G. B. Cerati, ${ }^{119}$ D. Evans, ${ }^{119}$ F. Golf, ${ }^{119}$ A. Holzner, ${ }^{119}$ R. Kelley, ${ }^{119}$ M. Lebourgeois, ${ }^{119}$ J. Letts, ${ }^{119}$ B. Mangano, ${ }^{119}$ S. Padhi, ${ }^{119}$ C. Palmer, ${ }^{119}$ G. Petrucciani, ${ }^{119}$ H. Pi, ${ }^{119}$ M. Pieri, ${ }^{119}$ R. Ranieri, ${ }^{119}$ M. Sani, ${ }^{119}$ V. Sharma, ${ }^{119}$ S. Simon, ${ }^{119}$ E. Sudano, ${ }^{119}$ M. Tadel, ${ }^{119}$ Y. Tu, ${ }^{119}$ A. Vartak, ${ }^{119}$ S. Wasserbaech, ${ }^{119, \text { ss }}$ F. Würthwein, ${ }^{119}$ A. Yagil, ${ }^{119}$ J. Yoo, ${ }^{119}$ D. Barge, ${ }^{120}$ R. Bellan, ${ }^{120}$ C. Campagnari, ${ }^{120}$ M. D’Alfonso, ${ }^{120}$ T. Danielson, ${ }^{120}$ K. Flowers, ${ }^{120}$ P. Geffert, ${ }^{120}$ J. Incandela, ${ }^{120}$ C. Justus, ${ }^{120}$ P. Kalavase, ${ }^{120}$ S. A. Koay, ${ }^{120}$ D. Kovalskyi, ${ }^{120}$ V. Krutelyov, ${ }^{120}$ S. Lowette, ${ }^{120}$ N. Mccoll, ${ }^{120}$ V. Pavlunin, ${ }^{120}$ F. Rebassoo, ${ }^{120}$ J. Ribnik, ${ }^{120}$ J. Richman, ${ }^{120}$ R. Rossin, ${ }^{120}$ D. Stuart, ${ }^{120}$ W. To, ${ }^{120}$ J. R. Vlimant, ${ }^{120}$ A. Apresyan, ${ }^{121}$ A. Bornheim, ${ }^{121}$ J. Bunn, ${ }^{121}$ Y. Chen, ${ }^{121}$ M. Gataullin, ${ }^{121}$ Y. Ma, ${ }^{121}$ A. Mott, ${ }^{121}$ H. B. Newman, ${ }^{121}$ C. Rogan, ${ }^{121}$ K. Shin, ${ }^{121}$ V. Timciuc, ${ }^{121}$ P. Traczyk, ${ }^{121}$ J. Veverka, ${ }^{121}$ R. Wilkinson, ${ }^{121}$ Y. Yang, ${ }^{121}$ R. Y. Zhu, ${ }^{121}$ B. Akgun, ${ }^{122}$ R. Carroll, ${ }^{122}$ T. Ferguson, ${ }^{122}$ Y. Iiyama, ${ }^{122}$ D. W. Jang, ${ }^{122}$ S. Y. Jun, ${ }^{122}$ Y. F. Liu, ${ }^{122}$

M. Paulini, ${ }^{122}$ J. Russ, ${ }^{122}$ H. Vogel, ${ }^{122}$ I. Vorobiev, ${ }^{122}$ J. P. Cumalat, ${ }^{123}$ M. E. Dinardo, ${ }^{123}$ B. R. Drell, ${ }^{123}$ C. J. Edelmaier, ${ }^{123}$ W. T. Ford, ${ }^{123}$ A. Gaz, ${ }^{123}$ B. Heyburn, ${ }^{123}$ E. Luiggi Lopez, ${ }^{123}$ U. Nauenberg, ${ }^{123}$ J. G. Smith, ${ }^{123}$ 
K. Stenson, ${ }^{123}$ K. A. Ulmer, ${ }^{123}$ S. R. Wagner, ${ }^{123}$ S. L. Zang, ${ }^{123}$ L. Agostino, ${ }^{124}$ J. Alexander, ${ }^{124}$ D. Cassel, ${ }^{124}$

A. Chatterjee, ${ }^{124}$ N. Eggert, ${ }^{124}$ L. K. Gibbons, ${ }^{124}$ B. Heltsley, ${ }^{124}$ K. Henriksson, ${ }^{124}$ W. Hopkins, ${ }^{124}$

A. Khukhunaishvili, ${ }^{124}$ B. Kreis, ${ }^{124}$ G. Nicolas Kaufman, ${ }^{124}$ J. R. Patterson, ${ }^{124}$ D. Puigh, ${ }^{124}$ A. Ryd,${ }^{124}$ M. Saelim, ${ }^{124}$ E. Salvati, ${ }^{124}$ X. Shi, ${ }^{124}$ W. Sun, ${ }^{124}$ W. D. Teo, ${ }^{124}$ J. Thom, ${ }^{124}$ J. Thompson, ${ }^{124}$ J. Vaughan, ${ }^{124}$ Y. Weng, ${ }^{124}$ L. Winstrom, ${ }^{124}$ P. Wittich, ${ }^{124}$ A. Biselli, ${ }^{125}$ G. Cirino, ${ }^{125}$ D. Winn, ${ }^{125}$ S. Abdullin, ${ }^{126}$ M. Albrow, ${ }^{126}$ J. Anderson, ${ }^{126}$ G. Apollinari, ${ }^{126}$ M. Atac, ${ }^{126}$ J. A. Bakken, ${ }^{126}$ L. A. T. Bauerdick, ${ }^{126}$ A. Beretvas, ${ }^{126}$ J. Berryhill, ${ }^{126}$ P. C. Bhat, ${ }^{126}$ I. Bloch, ${ }^{126}$ F. Borcherding, ${ }^{126}$ K. Burkett, ${ }^{126}$ J. N. Butler, ${ }^{126}$ V. Chetluru, ${ }^{126}$ H. W. K. Cheung, ${ }^{126}$ F. Chlebana, ${ }^{126}$ S. Cihangir, ${ }^{126}$ W. Cooper, ${ }^{126}$ D. P. Eartly, ${ }^{126}$ V. D. Elvira, ${ }^{126}$ S. Esen, ${ }^{126}$ I. Fisk, ${ }^{126}$ J. Freeman, ${ }^{126}$ Y. Gao, ${ }^{126}$ E. Gottschalk, ${ }^{126}$ D. Green, ${ }^{126}$ K. Gunthoti, ${ }^{126}$ O. Gutsche, ${ }^{126}$ J. Hanlon, ${ }^{126}$ R. M. Harris, ${ }^{126}$ J. Hirschauer, ${ }^{126}$ B. Hooberman, ${ }^{126}$ H. Jensen, ${ }^{126}$ M. Johnson, ${ }^{126}$ U. Joshi, ${ }^{126}$ R. Khatiwada, ${ }^{126}$ B. Klima, ${ }^{126}$ K. Kousouris, ${ }^{126}$ S. Kunori, ${ }^{126}$ S. Kwan, ${ }^{126}$ C. Leonidopoulos, ${ }^{126}$ P. Limon, ${ }^{126}$ D. Lincoln, ${ }^{126}$ R. Lipton, ${ }^{126}$ J. Lykken, ${ }^{126}$ K. Maeshima, ${ }^{126}$ J. M. Marraffino, ${ }^{126}$ D. Mason, ${ }^{126}$ P. McBride, ${ }^{126}$ T. Miao, ${ }^{126}$ K. Mishra, ${ }^{126}$ S. Mrenna, ${ }^{126}$ Y. Musienko, ${ }^{126, t \mathrm{tt}}$ C. Newman-Holmes, ${ }^{126}$ V. O’Dell, ${ }^{126}$ R. Pordes, ${ }^{126}$ O. Prokofyev, ${ }^{126}$ N. Saoulidou, ${ }^{126}$ E. Sexton-Kennedy, ${ }^{126}$ S. Sharma, ${ }^{126}$ W. J. Spalding, ${ }^{126}$ L. Spiegel, ${ }^{126}$ P. Tan, ${ }^{126}$ L. Taylor, ${ }^{126}$ S. Tkaczyk,${ }^{126}$ L. Uplegger, ${ }^{126}$ E. W. Vaandering, ${ }^{126}$ R. Vidal, ${ }^{126}$ J. Whitmore, ${ }^{126} \mathrm{~W}$. Wu, ${ }^{126} \mathrm{~F}$. Yang, ${ }^{126}$ F. Yumiceva ${ }^{126}$ J. C. Yun, ${ }^{126}$ D. Acosta, ${ }^{127}$ P. Avery, ${ }^{127}$ D. Bourilkov, ${ }^{127}$ M. Chen, ${ }^{127}$ S. Das,${ }^{127}$ M. De Gruttola, ${ }^{127}$

G. P. Di Giovanni, ${ }^{127}$ D. Dobur, ${ }^{127}$ A. Drozdetskiy, ${ }^{127}$ R. D. Field, ${ }^{127}$ M. Fisher, ${ }^{127}$ Y. Fu, ${ }^{127}$ I. K. Furic,${ }^{127}$ J. Gartner, ${ }^{127}$ J. Hugon, ${ }^{127}$ B. Kim, ${ }^{127}$ J. Konigsberg, ${ }^{127}$ A. Korytov, ${ }^{127}$ A. Kropivnitskaya, ${ }^{127}$ T. Kypreos, ${ }^{127}$ J. F. Low, ${ }^{127}$ K. Matchev, ${ }^{127}$ G. Mitselmakher, ${ }^{127}$ L. Muniz, ${ }^{127}$ C. Prescott, ${ }^{127}$ R. Remington, ${ }^{127}$ A. Rinkevicius, ${ }^{127}$ M. Schmitt, ${ }^{127}$ B. Scurlock, ${ }^{127}$ P. Sellers, ${ }^{127}$ N. Skhirtladze, ${ }^{127}$ M. Snowball, ${ }^{127}$ D. Wang,,${ }^{127}$ J. Yelton, ${ }^{127}$ M. Zakaria, ${ }^{127}$ V. Gaultney, ${ }^{128}$ L. M. Lebolo, ${ }^{128}$ S. Linn, ${ }^{128}$ P. Markowitz, ${ }^{128}$ G. Martinez, ${ }^{128}$ J. L. Rodriguez, ${ }^{128}$ T. Adams, ${ }^{129}$ A. Askew ${ }^{129}$ J. Bochenek, ${ }^{129}$ J. Chen, ${ }^{129}$ B. Diamond, ${ }^{129}$ S. V. Gleyzer, ${ }^{129}$ J. Haas, ${ }^{129}$ S. Hagopian, ${ }^{129}$ V. Hagopian, ${ }^{129}$ M. Jenkins, ${ }^{129}$ K. F. Johnson, ${ }^{129}$ H. Prosper, ${ }^{129}$ L. Quertenmont, ${ }^{129}$ S. Sekmen, ${ }^{129}$

V. Veeraraghavan, ${ }^{129}$ M. M. Baarmand, ${ }^{130}$ B. Dorney, ${ }^{130}$ S. Guragain, ${ }^{130}$ M. Hohlmann, ${ }^{130}$ H. Kalakhety, ${ }^{130}$ R. Ralich, ${ }^{130}$ I. Vodopiyanov, ${ }^{130}$ M. R. Adams, ${ }^{131}$ I. M. Anghel,${ }^{131}$ L. Apanasevich, ${ }^{131}$ Y. Bai, ${ }^{131}$ V. E. Bazterra, ${ }^{131}$ R. R. Betts, ${ }^{131}$ J. Callner, ${ }^{131}$ R. Cavanaugh, ${ }^{131}$ C. Dragoiu, ${ }^{131}$ L. Gauthier, ${ }^{131}$ C. E. Gerber, ${ }^{131}$ D. J. Hofman, ${ }^{131}$ S. Khalatyan, ${ }^{131}$ G. J. Kunde, ${ }^{131, \mathrm{vv}}$ F. Lacroix, ${ }^{131}$ M. Malek, ${ }^{131}$ C. O'Brien, ${ }^{131}$ C. Silkworth,${ }^{131}$ C. Silvestre, ${ }^{131}$ A. Smoron, ${ }^{131}$ D. Strom, ${ }^{131}$ N. Varelas, ${ }^{131}$ U. Akgun, ${ }^{132}$ E. A. Albayrak, ${ }^{132}$ B. Bilki, ${ }^{132}$ W. Clarida, ${ }^{132}$ F. Duru, ${ }^{132}$ C. K. Lae, ${ }^{132}$ E. McCliment, ${ }^{132}$ J.-P. Merlo, ${ }^{132}$ H. Mermerkaya, ${ }^{132, \text { uu }}$ A. Mestvirishvili, ${ }^{132}$ A. Moeller, ${ }^{132}$

J. Nachtman, ${ }^{132}$ C. R. Newsom, ${ }^{132}$ E. Norbeck, ${ }^{132}$ J. Olson, ${ }^{132}$ Y. Onel, ${ }^{132}$ F. Ozok, ${ }^{132}$ S. Sen, ${ }^{132}$ J. Wetzel, ${ }^{132}$ T. Yetkin, ${ }^{132}$ K. Yi, ${ }^{132}$ B. A. Barnett, ${ }^{133}$ B. Blumenfeld, ${ }^{133}$ A. Bonato, ${ }^{133}$ C. Eskew, ${ }^{133}$ D. Fehling, ${ }^{133}$ G. Giurgiu, ${ }^{133}$

A. V. Gritsan, ${ }^{133}$ Z. J. Guo, ${ }^{133}$ G. Hu, ${ }^{133}$ P. Maksimovic, ${ }^{133}$ S. Rappoccio, ${ }^{133}$ M. Swartz, ${ }^{133}$ N. V. Tran, ${ }^{133}$ A. Whitbeck, ${ }^{133}$ P. Baringer, ${ }^{134}$ A. Bean, ${ }^{134}$ G. Benelli, ${ }^{134}$ O. Grachov,${ }^{134}$ R. P. Kenny Iii, ${ }^{134}$ M. Murray, ${ }^{134}$ D. Noonan, ${ }^{134}$ S. Sanders, ${ }^{134}$ J. S. Wood, ${ }^{134}$ V. Zhukova, ${ }^{134}$ A. F. Barfuss, ${ }^{135}$ T. Bolton, ${ }^{135}$ I. Chakaberia, ${ }^{135}$ A. Ivanov, ${ }^{135}$ S. Khalil,,${ }^{135}$ M. Makouski, ${ }^{135}$ Y. Maravin, ${ }^{135}$ S. Shrestha, ${ }^{135}$ I. Svintradze, ${ }^{135}$ Z. Wan,,${ }^{135}$ J. Gronberg, ${ }^{136}$ D. Lange, ${ }^{136}$ D. Wright, ${ }^{136}$ A. Baden, ${ }^{137}$ M. Boutemeur, ${ }^{137}$ S. C. Eno, ${ }^{137}$ D. Ferencek, ${ }^{137}$ J. A. Gomez, ${ }^{137}$ N. J. Hadley, ${ }^{137}$ R. G. Kellogg, ${ }^{137}$ M. Kirn, ${ }^{137}$ Y. Lu, ${ }^{137}$ A. C. Mignerey, ${ }^{137}$ K. Rossato, ${ }^{137}$ P. Rumerio, ${ }^{137}$ F. Santanastasio, ${ }^{137}$ A. Skuja, ${ }^{137}$ J. Temple, ${ }^{137}$ M. B. Tonjes, ${ }^{137}$ S. C. Tonwar, ${ }^{137}$ E. Twedt,${ }^{137}$ B. Alver, ${ }^{138}$ G. Bauer, ${ }^{138}$ J. Bendavid, ${ }^{138}$ W. Busza, ${ }^{138}$ E. Butz,${ }^{138}$ I. A. Cali, ${ }^{138}$ M. Chan,${ }^{138}$ V. Dutta, ${ }^{138}$ P. Everaerts, ${ }^{138}$ G. Gomez Ceballos, ${ }^{138}$ M. Goncharov, ${ }^{138}$ K. A. Hahn, ${ }^{138}$ P. Harris, ${ }^{138}$ Y. Kim, ${ }^{138}$ M. Klute, ${ }^{138}$ Y.-J. Lee, ${ }^{138}$ W. Li ${ }^{138}$ C. Loizides, ${ }^{138}$ P. D. Luckey, ${ }^{138}$ T. Ma ${ }^{138}$ S. Nahn, ${ }^{138}$ C. Paus, ${ }^{138}$ D. Ralph, ${ }^{138}$ C. Roland,${ }^{138}$ G. Roland, ${ }^{138}$ M. Rudolph, ${ }^{138}$ G. S. F. Stephans, ${ }^{138}$ F. Stöckli, ${ }^{138}$ K. Sumorok, ${ }^{138}$ K. Sung,,${ }^{138}$ D. Velicanu, ${ }^{138}$ E. A. Wenger, ${ }^{138}$ S. Xie, ${ }^{138}$ M. Yang, ${ }^{138}$ Y. Yilmaz, ${ }^{138}$ A. S. Yoon, ${ }^{138}$ M. Zanetti, ${ }^{138}$ S. I. Cooper, ${ }^{139}$ P. Cushman, ${ }^{139}$ B. Dahmes, ${ }^{139}$ A. De Benedetti, ${ }^{139}$ P. R. Dudero, ${ }^{139}$ G. Franzoni, ${ }^{139}$ A. Gude, ${ }^{139}$ J. Haupt,${ }^{139}$ K. Klapoetke, ${ }^{139}$ Y. Kubota, ${ }^{139}$ J. Mans, ${ }^{139}$ N. Pastika, ${ }^{139}$ V. Rekovic, ${ }^{139}$ R. Rusack, ${ }^{139}$ M. Sasseville, ${ }^{139}$ A. Singovsky, ${ }^{139}$ N. Tambe, ${ }^{139}$ L. M. Cremaldi, ${ }^{140}$ R. Godang, ${ }^{140}$ R. Kroeger, ${ }^{140}$ L. Perera, ${ }^{140}$ R. Rahmat, ${ }^{140}$ D. A. Sanders,${ }^{140}$ D. Summers, ${ }^{140}$ K. Bloom, ${ }^{141}$ S. Bose, ${ }^{141}$ J. Butt, ${ }^{141}$ D. R. Claes, ${ }^{141}$ A. Dominguez, ${ }^{141}$ M. Eads, ${ }^{141}$ J. Keller, ${ }^{141}$ T. Kelly, ${ }^{141}$ I. Kravchenko, ${ }^{141}$ J. Lazo-Flores, ${ }^{141}$ H. Malbouisson, ${ }^{141}$ S. Malik, ${ }^{141}$ G. R. Snow, ${ }^{141}$ U. Baur, ${ }^{142}$ A. Godshalk, ${ }^{142}$ I. Iashvili, ${ }^{142}$ S. Jain, ${ }^{142}$ A. Kharchilava, ${ }^{142}$ A. Kumar, ${ }^{142}$ S. P. Shipkowski, ${ }^{142}$ K. Smith, ${ }^{142}$ J. Zennamo, ${ }^{142}$ G. Alverson, ${ }^{143}$ E. Barberis, ${ }^{143}$ D. Baumgartel, ${ }^{143}$ O. Boeriu, ${ }^{143}$ M. Chasco,${ }^{143}$ S. Reucroft, ${ }^{143}$ J. Swain, ${ }^{143}$ D. Trocino, ${ }^{143}$ D. Wood, ${ }^{143}$ J. Zhang, ${ }^{143}$ A. Anastassov, ${ }^{144}$ A. Kubik, ${ }^{144}$ N. Odell,,${ }^{144}$ 
R. A. Ofierzynski, ${ }^{144}$ B. Pollack, ${ }^{144}$ A. Pozdnyakov, ${ }^{144}$ M. Schmitt, ${ }^{144}$ S. Stoynev, ${ }^{144}$ M. Velasco, ${ }^{144}$ S. Won, ${ }^{144}$ L. Antonelli, ${ }^{145}$ D. Berry, ${ }^{145}$ A. Brinkerhoff, ${ }^{145}$ M. Hildreth, ${ }^{145}$ C. Jessop, ${ }^{145}$ D. J. Karmgard, ${ }^{145}$ J. Kolb, ${ }^{145}$ T. Kolberg, ${ }^{145}$ K. Lannon, ${ }^{145}$ W. Luo, ${ }^{145}$ S. Lynch, ${ }^{145}$ N. Marinelli, ${ }^{145}$ D. M. Morse, ${ }^{145}$ T. Pearson, ${ }^{145}$ R. Ruchti, ${ }^{145}$ J. Slaunwhite, ${ }^{145}$ N. Valls, ${ }^{145}$ M. Wayne, ${ }^{145}$ J. Ziegler, ${ }^{145}$ B. Bylsma, ${ }^{146}$ L. S. Durkin, ${ }^{146}$ J. Gu, ${ }^{146}$ C. Hill, ${ }^{146}$ P. Killewald, ${ }^{146}$ K. Kotov, ${ }^{146}$ T. Y. Ling, ${ }^{146}$ M. Rodenburg, ${ }^{146}$ G. Williams, ${ }^{146}$ N. Adam, ${ }^{147}$ E. Berry, ${ }^{147}$ P. Elmer, ${ }^{147}$ D. Gerbaudo, ${ }^{147}$ V. Halyo, ${ }^{147}$ P. Hebda, ${ }^{147}$ A. Hunt, ${ }^{147}$ J. Jones, ${ }^{147}$ E. Laird, ${ }^{147}$ D. Lopes Pegna, ${ }^{147}$ D. Marlow, ${ }^{147}$ T. Medvedeva, ${ }^{147}$ M. Mooney, ${ }^{147}$ J. Olsen, ${ }^{147}$ P. Piroué, ${ }^{147}$ X. Quan, ${ }^{147}$ B. Safdi, ${ }^{147}$ H. Saka, ${ }^{147}$ D. Stickland, ${ }^{147}$ C. Tully, ${ }^{147}$ J. S. Werner, ${ }^{147}$ A. Zuranski, ${ }^{147}$ J. G. Acosta, ${ }^{148}$ X. T. Huang, ${ }^{148}$ A. Lopez, ${ }^{148}$ H. Mendez, ${ }^{148}$ S. Oliveros, ${ }^{148}$ J. E. Ramirez Vargas, ${ }^{148}$ A. Zatserklyaniy, ${ }^{148}$ E. Alagoz, ${ }^{149}$ V. E. Barnes, ${ }^{149}$ G. Bolla, ${ }^{149}$ L. Borrello, ${ }^{149}$ D. Bortoletto, ${ }^{149}$ M. De Mattia, ${ }^{149}$ A. Everett, ${ }^{149}$ A. F. Garfinkel, ${ }^{149}$ L. Gutay, ${ }^{149}$ Z. Hu, ${ }^{149}$ M. Jones, ${ }^{149}$ O. Koybasi, ${ }^{149}$ M. Kress, ${ }^{149}$ A. T. Laasanen, ${ }^{149}$ N. Leonardo, ${ }^{149}$ C. Liu, ${ }^{149}$ V. Maroussov, ${ }^{149}$ P. Merkel, ${ }^{149}$ D. H. Miller, ${ }^{149}$ N. Neumeister, ${ }^{149}$ I. Shipsey, ${ }^{149}$ D. Silvers, ${ }^{149}$ A. Svyatkovskiy, ${ }^{149}$ H. D. Yoo, ${ }^{149}$ J. Zablocki, ${ }^{149}$ Y. Zheng, ${ }^{149}$ P. Jindal, ${ }^{150}$ N. Parashar, ${ }^{150}$ C. Boulahouache, ${ }^{151}$ K. M. Ecklund, ${ }^{151}$ F. J. M. Geurts, ${ }^{151}$ B. P. Padley, ${ }^{151}$ R. Redjimi, ${ }^{151}$ J. Roberts, ${ }^{151}$ J. Zabel, ${ }^{151}$ B. Betchart, ${ }^{152}$ A. Bodek, ${ }^{152}$ Y. S. Chung, ${ }^{152}$ R. Covarelli, ${ }^{152}$ P. de Barbaro, ${ }^{152}$ R. Demina, ${ }^{152}$ Y. Eshaq, ${ }^{152}$ H. Flacher, ${ }^{152}$ A. Garcia-Bellido, ${ }^{152}$ P. Goldenzweig, ${ }^{152}$ Y. Gotra, ${ }^{152}$ J. Han, ${ }^{152}$ A. Harel, ${ }^{152}$ D. C. Miner, ${ }^{152}$ D. Orbaker, ${ }^{152}$ G. Petrillo, ${ }^{152}$ W. Sakumoto, ${ }^{152}$ D. Vishnevskiy, ${ }^{152}$ M. Zielinski, ${ }^{152}$ A. Bhatti, ${ }^{153}$ R. Ciesielski, ${ }^{153}$ L. Demortier, ${ }^{153}$ K. Goulianos, ${ }^{153}$ G. Lungu, ${ }^{153} \mathrm{~S}$. Malik, ${ }^{153}$ C. Mesropian, ${ }^{153}$ O. Atramentov, ${ }^{154}$ A. Barker, ${ }^{154}$ D. Duggan, ${ }^{154}$ Y. Gershtein, ${ }^{154}$ R. Gray, ${ }^{154}$ E. Halkiadakis, ${ }^{154}$ D. Hidas, ${ }^{154}$ D. Hits, ${ }^{154}$ A. Lath, ${ }^{154}$ S. Panwalkar, ${ }^{154}$ R. Patel, ${ }^{154}$ K. Rose, ${ }^{154}$ S. Schnetzer, ${ }^{154}$ S. Somalwar, ${ }^{154}$ R. Stone, ${ }^{154}$ S. Thomas, ${ }^{154}$ G. Cerizza, ${ }^{155}$ M. Hollingsworth, ${ }^{155}$ S. Spanier, ${ }^{155}$ Z. C. Yang, ${ }^{155}$ A. York, ${ }^{155}$ R. Eusebi, ${ }^{156}$ W. Flanagan, ${ }^{156}$ J. Gilmore, ${ }^{156}$ A. Gurrola, ${ }^{156}$ T. Kamon, ${ }^{156}$ V. Khotilovich, ${ }^{156}$ R. Montalvo, ${ }^{156}$ I. Osipenkov, ${ }^{156}$ Y. Pakhotin, ${ }^{156}$ J. Pivarski, ${ }^{156}$ A. Safonov, ${ }^{156}$ S. Sengupta, ${ }^{156}$ A. Tatarinov, ${ }^{156}$ D. Toback, ${ }^{156}$ M. Weinberger, ${ }^{156}$ N. Akchurin, ${ }^{157}$ C. Bardak, ${ }^{157}$ J. Damgov, ${ }^{157}$ C. Jeong, ${ }^{157}$ K. Kovitanggoon, ${ }^{157}$ S. W. Lee, ${ }^{157}$ T. Libeiro, ${ }^{157}$ P. Mane, ${ }^{157}$ Y. Roh, ${ }^{157}$ A. Sill, ${ }^{157}$ I. Volobouev, ${ }^{157}$ R. Wigmans, ${ }^{157}$ E. Yazgan, ${ }^{157}$ E. Appelt, ${ }^{158}$ E. Brownson, ${ }^{158}$ D. Engh, ${ }^{158}$ C. Florez, ${ }^{158}$ W. Gabella, ${ }^{158}$ M. Issah, ${ }^{158}$ W. Johns, ${ }^{158}$ P. Kurt, ${ }^{158}$ C. Maguire, ${ }^{158}$ A. Melo, ${ }^{158}$ P. Sheldon, ${ }^{158}$ B. Snook, ${ }^{158}$ S. Tuo, ${ }^{158}$ J. Velkovska, ${ }^{158}$ M. W. Arenton, ${ }^{159}$ M. Balazs, ${ }^{159}$ S. Boutle, ${ }^{159}$ B. Cox, ${ }^{159}$ B. Francis, ${ }^{159}$ J. Goodell, ${ }^{159}$ R. Hirosky, ${ }^{159}$ A. Ledovskoy, ${ }^{159}$ C. Lin, ${ }^{159}$ C. Neu, ${ }^{159}$ R. Yohay, ${ }^{159}$ S. Gollapinni, ${ }^{160}$ R. Harr, ${ }^{160}$ P. E. Karchin, ${ }^{160}$ P. Lamichhane, ${ }^{160}$ M. Mattson, ${ }^{160}$ C. Milstène, ${ }^{160}$ A. Sakharov, ${ }^{160}$ M. Anderson, ${ }^{161}$ M. Bachtis, ${ }^{161}$ J. N. Bellinger, ${ }^{161}$ D. Carlsmith, ${ }^{161}$ S. Dasu, ${ }^{161}$ J. Efron, ${ }^{161}$ L. Gray, ${ }^{161}$ K. S. Grogg, ${ }^{161}$ M. Grothe ${ }^{161}$ R. Hall-Wilton, ${ }^{161}$ M. Herndon, ${ }^{161}$ A. Hervé, ${ }^{161}$ P. Klabbers, ${ }^{161}$ J. Klukas, ${ }^{161}$ A. Lanaro, ${ }^{161}$ C. Lazaridis, ${ }^{161}$ J. Leonard, ${ }^{161}$ R. Loveless, ${ }^{161}$ A. Mohapatra, ${ }^{161}$ F. Palmonari, ${ }^{161}$ D. Reeder, ${ }^{161}$ I. Ross, ${ }^{161}$ A. Savin, ${ }^{161}$ W. H. Smith, ${ }^{161}$ J. Swanson, ${ }^{161}$ and M. Weinberg ${ }^{161}$

\title{
(CMS Collaboration)
}

\author{
${ }^{1}$ Yerevan Physics Institute, Yerevan, Armenia \\ ${ }^{2}$ Institut für Hochenergiephysik der OeAW, Wien, Austria \\ ${ }^{3}$ National Centre for Particle and High Energy Physics, Minsk, Belarus \\ ${ }^{4}$ Universiteit Antwerpen, Antwerpen, Belgium \\ ${ }^{5}$ Vrije Universiteit Brussel, Brussel, Belgium \\ ${ }^{6}$ Université Libre de Bruxelles, Bruxelles, Belgium \\ ${ }^{7}$ Ghent University, Ghent, Belgium \\ ${ }^{8}$ Université Catholique de Louvain, Louvain-la-Neuve, Belgium \\ ${ }^{9}$ Université de Mons, Mons, Belgium \\ ${ }^{10}$ Centro Brasileiro de Pesquisas Fisicas, Rio de Janeiro, Brazil \\ ${ }^{11}$ Universidade do Estado do Rio de Janeiro, Rio de Janeiro, Brazil \\ ${ }^{12}$ Instituto de Fisica Teorica, Universidade Estadual Paulista, Sao Paulo, Brazil \\ ${ }^{13}$ Institute for Nuclear Research and Nuclear Energy, Sofia, Bulgaria \\ ${ }^{14}$ University of Sofia, Sofia, Bulgaria \\ ${ }^{15}$ Institute of High Energy Physics, Beijing, China \\ ${ }^{16}$ State Key Laboratory of Nuclear Physics and Technology, Peking University, Beijing, China \\ ${ }^{17}$ Universidad de Los Andes, Bogota, Colombia \\ ${ }^{18}$ Technical University of Split, Split, Croatia \\ ${ }^{19}$ University of Split, Split, Croatia
}




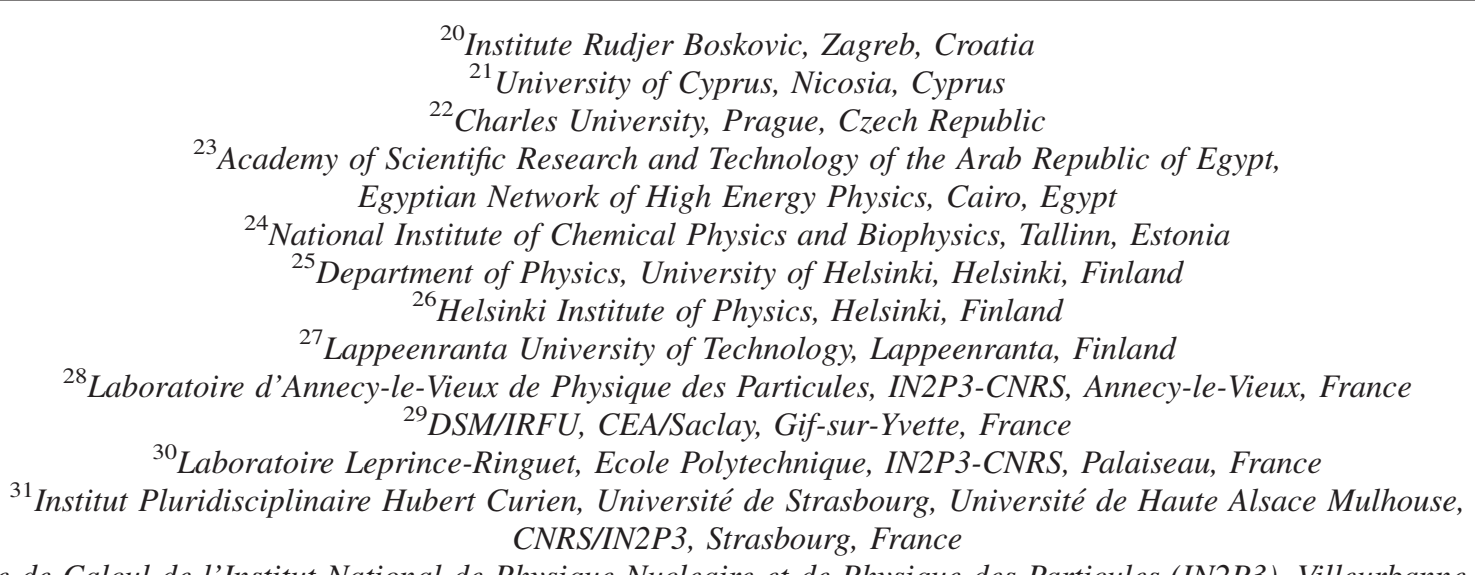

${ }^{32}$ Centre de Calcul de l'Institut National de Physique Nucleaire et de Physique des Particules (IN2P3), Villeurbanne, France

${ }^{33}$ Université de Lyon, Université Claude Bernard Lyon 1, CNRS-IN2P3, Institut de Physique Nucléaire de Lyon, Villeurbanne, France

${ }^{34}$ Institute of High Energy Physics and Informatization, Tbilisi State University, Tbilisi, Georgia

${ }^{35}$ RWTH Aachen University, I. Physikalisches Institut, Aachen, Germany

${ }^{36}$ RWTH Aachen University, III. Physikalisches Institut A, Aachen, Germany

${ }^{37}$ RWTH Aachen University, III. Physikalisches Institut B, Aachen, Germany

${ }^{38}$ Deutsches Elektronen-Synchrotron, Hamburg, Germany

${ }^{39}$ University of Hamburg, Hamburg, Germany

${ }^{40}$ Institut für Experimentelle Kernphysik, Karlsruhe, Germany

${ }^{41}$ Institute of Nuclear Physics “Demokritos,” Aghia Paraskevi, Greece

${ }^{42}$ University of Athens, Athens, Greece

${ }^{43}$ University of Ioánnina, Ioánnina, Greece

${ }^{44}$ KFKI Research Institute for Particle and Nuclear Physics, Budapest, Hungary

${ }^{45}$ Institute of Nuclear Research ATOMKI, Debrecen, Hungary

${ }^{46}$ University of Debrecen, Debrecen, Hungary

${ }^{47}$ Panjab University, Chandigarh, India

${ }^{48}$ University of Delhi, Delhi, India

${ }^{49}$ Saha Institute of Nuclear Physics, Kolkata, India

${ }^{50}$ Bhabha Atomic Research Centre, Mumbai, India

${ }^{51}$ Tata Institute of Fundamental Research-EHEP, Mumbai, India

${ }^{52}$ Tata Institute of Fundamental Research-HECR, Mumbai, India

${ }^{53}$ Institute for Research and Fundamental Sciences (IPM), Tehran, Iran

${ }^{54 a}$ INFN Sezione di Bari, Bari, Italy

${ }^{54 \mathrm{~b}}$ Università di Bari, Bari, Italy

${ }^{54 c}$ Politecnico di Bari, Bari, Italy

${ }^{55}$ INFN Sezione di Bologna, Bologna, Italy

${ }^{55 \mathrm{~b}}$ Università di Bologna, Bologna, Italy

${ }^{56 a}$ INFN Sezione di Catania, Catania, Italy

${ }^{56 \mathrm{~b}}$ Università di Catania, Catania, Italy

${ }^{57 a}$ INFN Sezione di Firenze, Firenze, Italy

${ }^{57 \mathrm{~b}}$ Università di Firenze, Firenze, Italy

${ }^{58}$ INFN Laboratori Nazionali di Frascati, Frascati, Italy

${ }^{59}$ INFN Sezione di Genova, Genova, Italy

${ }^{60 \mathrm{a}}$ INFN Sezione di Milano-Bicocca, Milano, Italy

${ }^{60 \mathrm{~b}}$ Università di Milano-Bicocca, Milano, Italy

${ }^{61 a}$ INFN Sezione di Napoli, Napoli, Italy

${ }^{61 \mathrm{~b}}$ Università di Napoli "Federico II," Napoli, Italy

${ }^{62 a}$ INFN Sezione di Padova, Padova, Italy

${ }^{62 \mathrm{~b}}$ Università di Padova, Padova, Italy

${ }^{62 \mathrm{c}}$ Università di Trento (Trento), Padova, Italy

${ }^{63}$ INFN Sezione di Pavia, Pavia, Italy

${ }^{63 \mathrm{~b}}$ Università di Pavia, Pavia, Italy

${ }^{64 \mathrm{a}}$ INFN Sezione di Perugia, Perugia, Italy

${ }^{64 \mathrm{~b}}$ Università di Perugia, Perugia, Italy

${ }^{65 a}$ INFN Sezione di Pisa, Pisa, Italy

${ }^{65 \mathrm{~b}}$ Università di Pisa, Pisa, Italy

${ }^{65}$ Scuola Normale Superiore di Pisa, Pisa, Italy 


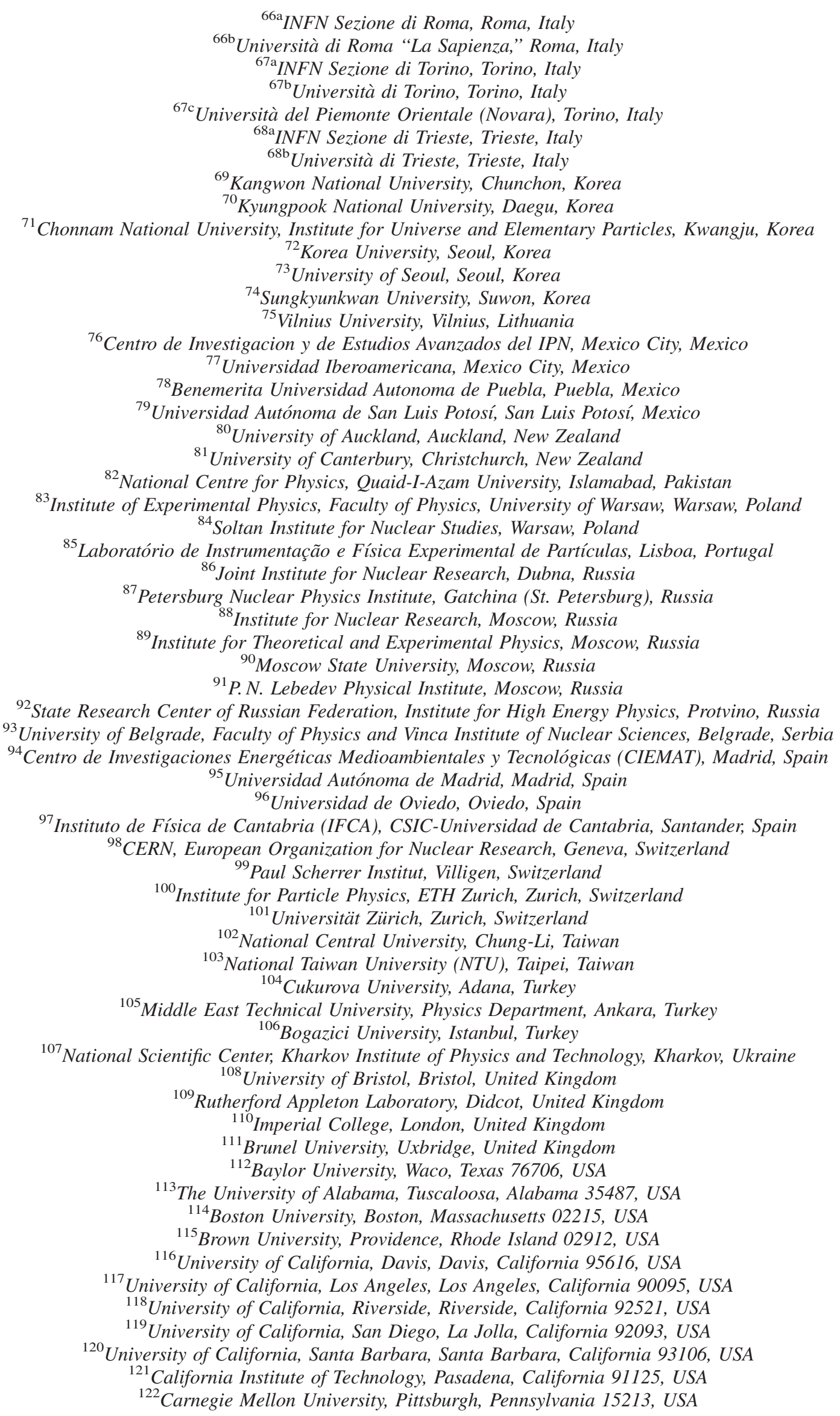




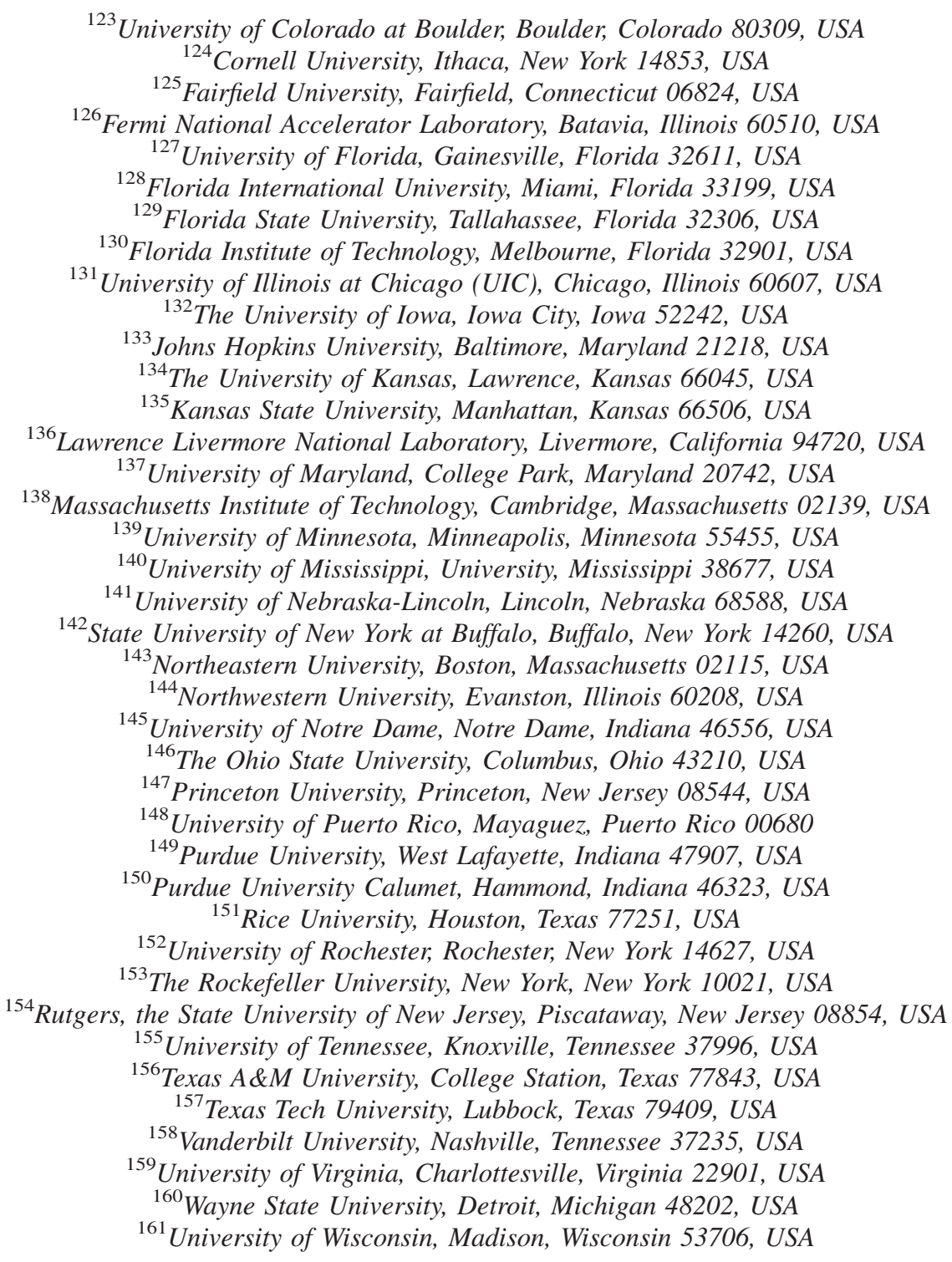

${ }^{\mathrm{a}}$ Deceased.

${ }^{\mathrm{b}}$ Also at CERN, European Organization for Nuclear Research, Geneva, Switzerland.

${ }^{\mathrm{c}}$ Also at Universidade Federal do ABC, Santo Andre, Brazil.

${ }^{\mathrm{d}}$ Also at Laboratoire Leprince-Ringuet, Ecole Polytechnique, IN2P3-CNRS, Palaiseau, France.

${ }^{\mathrm{e}}$ Also at Suez Canal University, Suez, Egypt.

${ }^{\mathrm{f}}$ Also at British University, Cairo, Egypt.

${ }^{\mathrm{g}}$ Also at Fayoum University, El-Fayoum, Egypt.

${ }^{\mathrm{h}}$ Also at Soltan Institute for Nuclear Studies, Warsaw, Poland.

${ }^{\mathrm{i}}$ Also at Massachusetts Institute of Technology, Cambridge, MA, USA.

${ }^{j}$ Also at Université de Haute-Alsace, Mulhouse, France.

${ }^{\mathrm{k}}$ Also at Brandenburg University of Technology, Cottbus, Germany.

${ }^{1}$ Also at Moscow State University, Moscow, Russia.

${ }^{\mathrm{m}}$ Also at Institute of Nuclear Research ATOMKI, Debrecen, Hungary.

${ }^{\mathrm{n}}$ Also at Eötvös Loránd University, Budapest, Hungary.

${ }^{\circ}$ Also at Tata Institute of Fundamental Research-HECR, Mumbai, India.

${ }^{\mathrm{p}}$ Also at University of Visva-Bharati, Santiniketan, India.

${ }^{\mathrm{q}}$ Also at Sharif University of Technology, Tehran, Iran.

${ }^{\mathrm{r}}$ Also at Shiraz University, Shiraz, Iran.

${ }^{\mathrm{s}}$ Also at Isfahan University of Technology, Isfahan, Iran. 
tAlso at Facoltà Ingegneria Università di Roma "La Sapienza,” Roma, Italy.

"Also at Università della Basilicata, Potenza, Italy.

${ }^{\mathrm{v}}$ Also at Università degli studi di Siena, Siena, Italy.

${ }^{w}$ Also at Faculty of Physics of University of Belgrade, Belgrade, Serbia.

${ }^{\mathrm{x}}$ Also at University of California, Los Angeles, Los Angeles, CA, USA.

${ }^{y}$ Also at University of Florida, Gainesville, FL, USA.

${ }^{\mathrm{z}}$ Also at Université de Genève, Geneva, Switzerland.

${ }^{\text {aa } A l s o ~ a t ~ S c u o l a ~ N o r m a l e ~ e ~ S e z i o n e ~ d e l l ' ~ I N F N, ~ P i s a, ~ I t a l y . ~}$

${ }^{\mathrm{bb}}$ Also at University of Athens, Athens, Greece.

${ }^{\mathrm{cc}}$ Also at California Institute of Technology, Pasadena, CA, USA.

${ }^{\mathrm{dd}}$ Also at The University of Kansas, Lawrence, KS, USA.

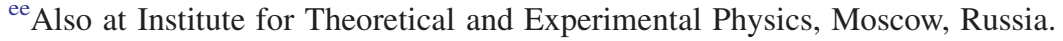

${ }^{\mathrm{ff}}$ Also at Paul Scherrer Institut, Villigen, Switzerland.

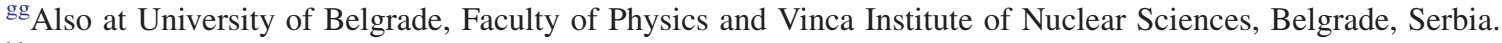

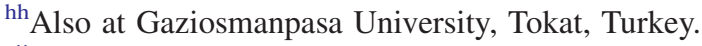

${ }^{i i}$ Also at Adiyaman University, Adiyaman, Turkey.

${ }^{\mathrm{jj}}$ Also at The University of Iowa, Iowa City, IA, USA.

${ }^{\mathrm{kk}} \mathrm{Also}$ at Mersin University, Mersin, Turkey.

${ }^{11}$ Also at Izmir Institute of Technology, Izmir, Turkey.

${ }^{\mathrm{mm}}$ Also at Kafkas University, Kars, Turkey.

${ }^{\mathrm{nn}}$ Also at Suleyman Demirel University, Isparta, Turkey.

${ }^{\circ o}$ Also at Ege University, Izmir, Turkey.

${ }^{\mathrm{pp}}$ Also at Rutherford Appleton Laboratory, Didcot, U.K.

${ }^{\mathrm{qq}}$ Also at School of Physics and Astronomy, University of Southampton, Southampton, U.K.

${ }^{\mathrm{rr}}$ Also at INFN Sezione di Perugia, Università di Perugia, Perugia, Italy.

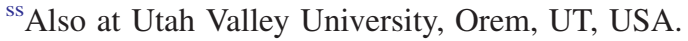

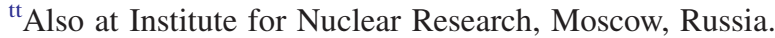

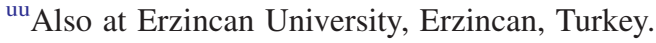

${ }^{\mathrm{vv}}$ Also at Los Alamos National Laboratory, Los Alamos, NM, USA. 